\title{
Evolution of Manganese-Nickel-Silicon Dominated Phases in Highly Irradiated Reactor Pressure Vessel Steels
}

\author{
Peter B. Wells ${ }^{1}$, Takuya Yamamoto ${ }^{1}$, Brandon Miller ${ }^{2}$, Tim Milot ${ }^{1}$, James Cole ${ }^{2}$, Yuan $\mathrm{Wu}^{1}$, \\ G. Robert Odette ${ }^{1}$ \\ 1. University of California, Santa Barbara, CA 93106-5050 \\ 2. Idaho National Laboratory, 2525 Fremont Ave. Idaho Falls, ID 83402
}

\section{Corresponding Author}

\section{Peter Wells}

PhD Candidate UCSB Materials Department

Email: pwells@umail.ucsb.edu

Cell: 208-403-9204

Address:

Materials Department

University of California

Santa Barbara, CA 93106-5050 


\begin{abstract}
Formation of a high density of $\mathrm{Mn}-\mathrm{Ni}-\mathrm{Si} \mathrm{nm}$-scale precipitates in irradiated $\mathrm{Cu}$-free and $\mathrm{Cu}-$ bearing reactor pressure vessel steels could lead to severe unexpected embrittlement. Models long ago predicted that these precipitates, which are not treated in current embrittlement prediction models, would emerge only at high fluence. However, the mechanisms and variables that control Mn-Ni-Si precipitate formation, and their detailed characteristics, have not been well understood. High flux irradiations of six steels with systematic variations in $\mathrm{Cu}$ and $\mathrm{Ni}$ contents were carried out at $\approx 295^{\circ} \mathrm{C}$ to high and very high neutron fluences of $\approx$ $1.3 \times 10^{20}$ and $1.1 \times 10^{21} \mathrm{n} / \mathrm{cm}^{2}$. Atom probe tomography (APT) shows that significant mole fractions of $\mathrm{Mn}-\mathrm{Ni}-\mathrm{Si}$ dominated precipitates form in the $\mathrm{Cu}$-bearing steels at $\approx 1.3 \times 10^{20}$ $\mathrm{n} / \mathrm{cm}^{2}$, while they are only beginning to develop in $\mathrm{Cu}$-free steels. However, large mole fractions of these precipitates, far in excess of those found in previous studies, are observed at $1.1 \times 10^{21} \mathrm{n} / \mathrm{cm}^{2}$ at all $\mathrm{Cu}$ contents. At the highest fluence, the precipitate mole fractions primarily depend on the alloy $\mathrm{Ni}$, rather than $\mathrm{Cu}$, content. The $\mathrm{Mn}-\mathrm{Ni}-\mathrm{Si}$ precipitates lead to very large increases in measured hardness, corresponding to yield strength elevations of up to almost $700 \mathrm{MPa}$.
\end{abstract}

Keywords: Radiation Damage; Atom Probe Tomography; Precipitation; Irradiation Embrittlement 


\section{Introduction}

Light water reactor (LWR) plant life extension to 80 years is needed to sustain the largest US carbon-free energy resource [1]. The major permanent and safety-critical LWR component is the massive reactor pressure vessel (RPV). Neutron irradiation of RPV steels results in embrittlement, manifested as upward brittle to ductile transition temperature shifts (TTS). TTS are primarily caused by the precipitation and defect hardening that occur under irradiation. Life extension requires demonstration that RPVs operate within safety margins, including ensuring that the effects of long-term irradiation on fracture toughness can be predicted and safely managed. The major RPV challenge for life extension is to develop robust predictive models of TTS in neutron flux and fluence regimes where data largely do not currently exist. Here we verify the existence of a potentially severe precipitation hardening and embrittlement mechanism that emerges only at high fluence. Notably, this mechanism is not accounted for in current embrittlement prediction models. We also report on a number of new insights and related details regarding the nature and potential impact of the responsible precipitation hardening phases.

Current US TTS regulations derive from physically motivated models fit to the in-service power reactor, engineering (surveillance) database [2-4]. These models treat the accelerated formation of $\mathrm{Cu}$-rich precipitates (CRPs), due to radiation-enhanced diffusion [5], as well as solute-defect cluster complexes, referred to here as matrix features, which form in steels both with and without significant $\mathrm{Cu}$ contents. Matrix features are believed to initially form in displacement cascades as defect-solute cluster complexes, or their defect-free remnants [2-4, 6-9]. Both CRP and matrix features also generally contain significant quantities of $\mathrm{Mn}, \mathrm{Ni}$ 
and Si [6-27]. However, current models predict that the CRP contribution to hardening saturates when $\mathrm{Cu}$ is depleted from the matrix [2,3]. Additional hardening due to matrix features increases linearly with the square root of fluence at the same relatively slow rate in both the $\mathrm{Cu}$-bearing and $\mathrm{Cu}$-free steels, and does not saturate. The semi-empirical models were fitted to the surveillance database that contained little high fluence $\left(>5 \times 10^{19} \mathrm{n} / \mathrm{cm}^{2}\right)$ TTS, pertinent to extended life. Thus extrapolations of TTS to 80-year operation may not be reliable [4].

Most notably, the potential for large mole fractions of Mn-Ni-Si (MNS) precipitates to form at high fluence is not included in the existing embrittlement models. This deficiency may be one source of large under-predictions of the TTS at high fluence observed in higher flux and fluence test reactor irradiations [4]. MNS phases are also of interest in their own right, since they would not be observed at low temperatures, around $300^{\circ} \mathrm{C}$, absent radiation enhanced diffusion that greatly accelerates their otherwise sluggish thermal kinetics. Further, the MNS precipitates, and the role of $\mathrm{Cu}$ in their formation, may point to new directions for high strength alloy development.

Unfortunately, with regard to RPV embrittlement, there are no in-service, low flux-high fluence data for 80-year in-service vessel conditions. Previous research first predicted, and subsequently confirmed, that MNS precipitates can form in low-Cu steels and that they likely emerge from the matrix feature solute-defect cluster complexes, cited above that begin forming at low fluence $[6-9,13,25]$. More recent studies by others have also observed MNS precipitates in other low- $\mathrm{Cu}$, high-Ni content steels, including in surveillance programs [17, 26, 27]. Large mole fractions of MNS precipitates are enhanced at higher Ni content and fluence as well as by lower irradiation temperatures and flux $[4,7,8,15]$. 
Here we focus on atom probe tomography (APT) studies of precipitates formed at two very high flux and high to very high fluence irradiation conditions. The experiments were carried out in the Belgian Reactor 2 (BR2) and the US Advanced Test Reactor (ATR), respectively. The test reactor fluxes range from 2500 (BR2) to 5800 (ATR) times higher than those in a vessel that reaches an end of life fluence of $\approx 10^{20} \mathrm{n} / \mathrm{cm}^{2}$ in 80 years. The corresponding BR2 and ATR fluence levels range from $\approx 1.3 \times 10^{20} \mathrm{n} / \mathrm{cm}^{2}$ to a previously unexplored $11 \times 10^{20} \mathrm{n} / \mathrm{cm}^{2}$.

As shown below, and for the first time, the very high fluence condition in this study produces extremely large mole fractions of MNS phases in six steels with systematic variations in their $\mathrm{Cu}$ and $\mathrm{Ni}$ contents. The effects of high flux will be discussed in detail in future publications. We note, however, that it is well known that higher flux affects hardening and embrittlement, primarily by delaying precipitation to higher fluence $[2,6-8$, 14, 28, 29]. This is illustrated in Figures $1 \mathrm{a}$ and $1 \mathrm{~b}$ for irradiation hardening, represented by an equivalent change in yield stress $\left(\Delta \sigma_{\mathrm{y}}\right)$ and precipitate volume fractions $\left(\mathrm{f}_{\mathrm{v}}\right)$ in a high $\mathrm{Cu}$, medium Ni content steel (LC), plotted as a function of the square root of fluence ( $\phi \mathrm{t})$, for low flux $(\phi)$ irradiations in the previous UCSB Irradiation Variables (IVAR) Program and high flux BR2 irradiations [29]. The increasing delay in both precipitation and hardening with increasing flux is obvious. These results are presented here simply to show that the precipitation hardening in these high to very high fluence test reactor irradiation conditions would likely occur at much lower fluence under low flux power reactor conditions. The fluxadjusted power reactor equivalent fluences for these irradiations are yet to be verified; but based on our current best estimates, the BR2 irradiation would likely have an equivalent fluence that occurs before an 80 year end of vessel service life, while the corresponding ATR 
irradiation equivalent fluence would require more than 80 years [29]. However, the data from the ATR irradiation permit interpolation to an 80 year condition, and provide insight about the detailed nature of MNS precipitates, are as well as how and why they form.

A description of the materials and methods is follow by a section that first summarizes data from the high fluence BR2 irradiation, followed by the corresponding data for the very high fluence ATR condition. Assessing similarities and differences between these two irradiation conditions provides insight, not only on MNS precipitation kinetics, but also on the sequence of events leading to the formation of large mole fractions of precipitates. Further, the very high fluence ATR irradiation produces precipitates that are well developed, at both larger sizes and mole fractions than have been previously observed. Thus these data are analyzed in great detail, since the larger MNS precipitates are more amenable to detailed characterization by APT. The paper concludes with a brief discussion of some of the implication of our observations, a summary of the main conclusions and a few comments on future work.

\section{Experimental}

\subsection{Materials and Irradiation Conditions}

A large number of RPV split melt pressure vessel steels were irradiated to a wide range of irradiation conditions in IVAR, BR2, and the ATR. This paper will present data for only six steels in two of the irradiation conditions, which are shown in Table 1. The first irradiation was carried out at a flux of $\approx 1.0 \times 10^{14} \mathrm{n} / \mathrm{cm}^{2}$-s to a high fluence of $\approx 1.3 \times 10^{20}$ $\mathrm{n} / \mathrm{cm}^{2}$ in $\mathrm{BR} 2$ at $\approx 300^{\circ} \mathrm{C}$. The second irradiation was carried out at a flux of $\approx 2.3 \times 10^{14}$ $\mathrm{n} / \mathrm{cm}^{2}$-s to a very high fluence of $1.1 \times 10^{21} \mathrm{n} / \mathrm{cm}^{2}$ in ATR at $\approx 290^{\circ} \mathrm{C}$. The high fluence BR2 specimens were in the form of $23 \times 3 \times 0.5 \mathrm{~mm}$ coupons. The very high fluence specimens in 
the ATR irradiation were in the form of $8 \times 0.5 \mathrm{~mm}$ discs. Four steels with similar $\mathrm{Ni}(\approx 0.8$ wt.\%), $\mathrm{Mn}(\approx 1.4$ wt. $\%)$ and $\mathrm{Si}(\approx 0.23$ wt. $\%)$ contents had systematic variations in their bulk $\mathrm{Cu}$ content from 0.01 to 0.41 wt.\% (LG, LH, LI, LC). One high $\mathrm{Cu}$ content steel (LD) had a larger 1.25 wt.\% Ni, while another, that was essentially $\mathrm{Cu}$-free, contained the highest 1.68 wt.\% Ni along with 1.50 wt.\% Mn and lower $0.17 \%$ Si (CM6). The steel compositions and heat treatments are summarized in Table 2 . As a terminology guide, alloys that contain $\approx$ $0.8 \% \mathrm{Ni}$ (LG, LH, LI, LC) are referred to as medium Ni content steels, the alloy with $1.25 \%$ $\mathrm{Ni}$ and $0.38 \% \mathrm{Cu}(\mathrm{LD})$ is referred to as the high $\mathrm{Ni}-\mathrm{Cu}$ content steel, and the $\mathrm{Cu}$-free alloy with $1.68 \% \mathrm{Ni}$ (CM6) is referred to as the highest $\mathrm{Ni}$ content, $\mathrm{Cu}$-free steel.

\subsection{Characterization Techniques}

A wide variety of microstructural and mechanical property characterization methods are being applied in post-irradiation evaluations of these high to very high fluence irradiation conditions. As dictated by the specimen size, microhardness was used to measure strength increases, and atom probe tomography (APT) was used to characterize the precipitates. The discussion, which follows, focuses on APT methods and issues.

\subsubsection{Atom Probe Tomography (APT)}

APT on the high fluence BR2 steels was carried out at the University of California, Santa Barbara (UCSB) on a Cameca LEAP 3000X HR, while the very high fluence ATR steels were examined using a Cameca LEAP 4000X HR at the Idaho Center for Advanced Energy Studies (CAES). Both instruments are equipped with an energy compensating reflectron, which improves mass resolution. APT tips were fabricated by focused ion beam milling (FIBing) at CAES and the University of California, Berkeley. A low energy cleanup at $2 \mathrm{kV}$ was used to reduce the Ga damage and contamination of the tips. 
A complete discussion of APT procedures and issues are beyond the scope of this paper. More detailed coverage of these topics can partly be found in [30, 31]. The main APT analysis parameters and improvements with respect to typical practice are summarized below.

All samples were run in voltage mode at a temperature of $50 \mathrm{~K}$ with a pulse fraction of $20 \%$, a pulse repetition rate of $200 \mathrm{kHz}$ and target evaporation rate between $0.5 \%$ and $1 \%$ per pulse. Mass to charge state ratio peak deconvolution was performed on overlapping element peaks, using the natural abundances of the various isotopes for individual elements with nonoverlapping peaks [30]. The tip radius as a function of depth was estimated using SEM images taken after the final FIB milling step. This information was used in the tip profile reconstruction method, included in the Cameca Integrated Visualization and Analysis (IVAS) commercial software, instead of estimating the radius from the voltage evolution and $\mathrm{k}$ factor. The first several hundred thousand atoms were typically excluded from the reconstruction because these ions evaporated as the tip was being aligned and had a higher Ga concentration than is preferred. Excluding these atoms meant that the initial tip radius for the reconstruction was selected based on enforcing plane spacing criteria for low index poles [(110), (200) or both].

The cluster analysis was performed using a modified version of the maximum separation method, also referred to as the density-based clustering algorithm; here, instead of finding the distance (d) between a solute, defined as $\mathrm{Cu}, \mathrm{Ni}, \mathrm{Mn}$, and $\mathrm{Si}$, and its nearest solute neighbor, the corresponding distance between a solute and its $5^{\text {th }}$ nearest solute neighbor is found [32]. If $\mathrm{d}$ is less than a defined distance $\left(\mathrm{d}_{\max }\right)$, the solute is considered to be a core atom. After all core atoms have been found, any solute atom within $\mathrm{d}_{\max }$ of a core atom is considered to be in 
the same cluster. Any cluster with less than $\mathrm{N}_{\min }$ atoms is excluded from the analysis. We used $\mathrm{d}_{\max }=0.50-0.60 \mathrm{~nm}$ and $\mathrm{N}_{\min }=15-30$ in our analysis.

APT results must be interpreted cautiously in the face of a number of potential artifacts of the technique. Lower evaporation fields, associated with more weakly bound precipitate atoms, result in flattening, or even dimpling, of the region in the vicinity of the feature. This, in turn, produces tip topology-induced ion trajectory aberrations and changes in the local magnification factors, specifying the separations and densities of atoms at the detector versus the actual physical tip. In this case, artifact matrix atoms are focused into the precipitate region of the detector along with the solute atoms [33]. These so-called trajectory aberrations result in artifacts in the apparent composition of the precipitates, and are signaled by higherthan-physical local atom densities. Further, coupled with assumptions used in the reconstruction algorithms, in some cases spatially inhomogeneous evaporation sequences may also lead to distortion of the shapes of reconstructed precipitates (flattening or elongation), as well as features with chemically segregated tops and/or bottoms (when there are no physical tops and bottoms along the APT analysis z-direction) [33-35].

These APT issues have long been recognized, but have not yet been resolved. However, they are of current intense interest, and recent research is leading to much better understanding of APT artifacts; for example, electrostatic simulations of field emission from a tip containing small features with a range of evaporation fields have been combined with experimental observations of spatial-temporal correlations in atom emission sequence [35]. The most relevant conclusion is that excess Fe in precipitates is almost certainly largely an APT artifact, if only because the atom densities in the precipitates are 2-3 times higher than is physical. This conclusion is reinforced by the fact that high Fe content is inconsistent with 
other techniques, such as small angle neutron scattering (SANS), as only one of several examples, as well as with thermodynamic considerations $[8,16]$. The high Fe content in the MNS precipitates, that are the focus of this study, is also inconsistent with first principles modeling of the $\mathrm{Mn}_{6} \mathrm{Ni}_{16} \mathrm{Si}_{7} \mathrm{G}$ phase, since this indicates that $\mathrm{Fe}$ would add $\approx 0.3-0.4$ eV/atom [36]. Thus our analysis assumes the precipitates found in this study do not contain a significant amount of Fe. However, the nominal Fe contents are included in the tables summarizing the precipitate compositions for those that choose to interpret the data differently.

The precipitate sizes were calculated by determining the spherical radius, $r_{p}$, of the volume of total number of solute atoms in a cluster, corrected for the detection efficiency, assuming the solute atomic volume that is the same as for Fe, as $r_{p}=\sqrt[3]{\frac{3 N_{\text {sol }} \Omega}{4 \pi \eta}}$. Here $\mathrm{N}_{\text {sol }}$ is the number of $\mathrm{Cu}, \mathrm{Ni}, \mathrm{Mn}$, and $\mathrm{Si}$ atoms in a given cluster, $\Omega$ is the atomic volume of $\mathrm{Fe}$, and $\eta$ is the efficiency of the LEAP (0.37 in the case of a reflectron-equipped instrument). The possible intermetallic phases that exist in these steels have atom densities that vary from those of Fe within $\pm 10 \%$, so the corresponding effect on $r_{p}$ is less than about $\pm 3 \%$. Given the various uncertainties in the APT reconstructions, we believe that this method of estimating $r_{p}$ is more physically justified and systematic compared to other common approaches, like multi-axis Guiner radius based estimates, especially since the artifacts discussed above leave the precipitates with distorted shapes, length scales and atomic densities $[20,33,35]$. The corresponding cluster mole fraction, $\mathrm{f}$, is estimated by dividing the total number of solutes in the precipitates by the total number of atoms in the sample.

Precipitates on the edge of the reconstruction are not used in calculating the size distributions. However, edge precipitates are counted as one half in determining their total 
number density, $\mathrm{N}$. The $\mathrm{N}$ is calculated by dividing the total number of clusters by the total reconstruction volume, which is determined by multiplying the total number of atoms in the reconstruction, corrected for the efficiency, by the atomic volume of Fe. There is also a possibility of over estimating the residual solutes left in the matrix due to the presence of trajectory aberrations $[35,37,38]$. To account for this, the matrix atoms immediately surrounding the clusters are excluded from the matrix composition.

Multiple APT measurements were carried out on each alloy, except in two cases where only one tip was available. The mean bulk, matrix and precipitate compositions, as well as precipitate $\langle\mathrm{r}\rangle, \mathrm{N}$ and $\mathrm{f}$ were determined for each tip for a given alloy nominal condition. The ion weighted mean and standard deviation of the measurements were calculated for a given alloy from the means of all runs. The expression for the weighted mean and weighted standard deviation are given by

$$
\begin{gathered}
\bar{x}=\frac{\sum_{i=1}^{n} w_{i} x_{i}}{\sum_{i=1}^{n} w_{i}} \\
S=\sqrt{\frac{n}{(n-1)} * \frac{\sum_{i=1}^{n} w_{i}\left(x_{i}-\bar{x}\right)^{2}}{\sum_{i=1}^{n} w_{i}}}
\end{gathered}
$$

Here $\bar{x}$ is the mean value for a set of given alloy condition runs, $\mathrm{x}_{\mathrm{i}}$ is the calculated value for the i'th run for a single alloy condition, $S$ is the standard deviation of the means for the alloy, $\mathrm{w}_{\mathrm{i}}$ is the total number of atoms for the $\mathrm{i}$ th measurement, and $\mathrm{n}$ is the total number of measurements on a single alloy condition. The mean value uncertainties were taken as the standard error of a given quantity as $S E=\frac{S}{\sqrt{n}}$.

In two cases at high fluence, the medium $\mathrm{Ni}$, high $\mathrm{Cu}$ content (LC) and highest $\mathrm{Ni}$ content, $\mathrm{Cu}$-free (CM6) alloys, when only one APT tip was available, the uncertainty was 
estimated using the uncertainty for other alloys, with similar compositions, but increased by a factor of two. These estimated uncertainties are flagged in the tables with an asterisk.

The main source of analysis uncertainty in calculating $\mathrm{f}$ for a single run is the selection of $d_{\max }$ and $N_{\min }$. A parametric study was performed varying $d_{\max }$ by $\pm 10 \%$. The corresponding $f$ varied by $\approx \pm 5 \%$ of the total and the individual $\mathrm{Ni}, \mathrm{Mn}$ and $\mathrm{Si}$ varied by $\approx \pm 6 \%$, while the $\mathrm{Cu}$ showed very little variation. These uncertainties in $\mathrm{f}$ for single tips were used in the scatter plots of $f$ vs. bulk composition for individual runs.

Finally, a major general limitation of APT is the very small volumes of material that are probed, typically on the order of $10^{-16} \mathrm{~cm}^{3}$. This is a critical issue for heterogeneously distributed features, especially if their number densities are low. While the precipitates in these steels are not completely homogeneously distributed, the results show very similar high number densities in all the steels with similar Ni content. Additionally, APT samples were made from two separate liftout locations for three of the steels (LC, LD, and CM6) in the very high dose condition; both locations showed consistent precipitate sizes and number densities for a given steel. This relatively uniform distribution of precipitates allowed us to use compositional heterogeneities to advantage. Specifically, tip-to-tip variations in the overall local bulk solute compositions, which were significant in some cases, can be directly correlated with the corresponding compositions and $\mathrm{f}$ of the precipitates on a nearly one-toone basis. We show below that the effects of tip-to-tip bulk composition variations for the same alloy condition are generally consistent with larger alloy-to-alloy bulk compositional variations with respect to their effects on the precipitate parameters. Consequently, all of the APT bulk compositions that follow are specified as either the actual measured values in at.\% for a given tip, or the average measured value for a given alloy condition. 
In closing this section we note that APT reconstructions contain a great amount of detailed information that is generally not available using other techniques. This includes associations between different features, core-shell structures, co-formation of different precipitates, relations between precipitate size and composition, and so on. The results described below give some examples of how such information can be exploited, but many additional opportunities can, and should be, explored in the future.

\subsubsection{Microhardness}

Microhardness tests were performed at UCSB on a Leco M400 hardness tester with at least 10 indents per sample at a load of 500 grams. A common calibration block was used in all cases to ensure the accuracy of the test. The mean and standard error were calculated for the hardness of each alloy in both the baseline and irradiated conditions. For the high flux irradiations, the as-irradiated hardness was taken both before and after a mild post-irradiation anneal at $350^{\circ} \mathrm{C}$ for $5 \mathrm{~h}$ to remove the thermally unstable damage that is likely associated only with high flux $[4,14,39]$. The yield stress increase was estimated using the relation $\Delta \sigma_{\mathrm{y}}$ $\approx 3.3 \Delta \mathrm{H}_{\mathrm{v}}[40]$. The uncertainty in the $\Delta \sigma_{\mathrm{y}}$ was calculated from the root mean square of the standard errors of the baseline and as-irradiated measurements.

\section{Results and Discussion}

We first describe the APT measurements of the total solute contents of the alloys in this study, and how they vary from nominal "bulk" chemistry values. These measurements show that the total APT and nominal bulk values are relatively consistent, differing only in ways that are expected. Next, we summarize the results for the high fluence irradiation condition where MNS precipitates are just beginning to be observed in the Cu-free steels (LG and CM6), likely representing a continued evolution of matrix feature clusters that are 
continuously formed in displacement cascades. Data from the other steels clearly show that $\mathrm{Cu}$ catalyzes MNS precipitate formation. We next show that this initial phase formation is followed by continued precipitation of the Mn-Ni-Si solutes up to a large fraction of the alloy contents of these elements. The large precipitate mole fractions observed at very high fluence, range from 1.3 to $2.8 \%$, and lead to correspondingly large equivalent $\Delta \sigma_{\mathrm{y}}$. Note that at very high fluence, the $\mathrm{Cu}$ fraction in the $\mathrm{Mn}-\mathrm{Ni}-\mathrm{Si}$ dominated precipitates ranges from $\approx 0$ to $15 \%$, in rough proportion to the alloy content of this element. At very high fluence, the $\mathrm{Cu}$ is nearly fully depleted from the matrix. The well-established precipitates at very high fluence provide a basis for more detailed characterization and analysis of their dependence on the local alloy composition and various morphological details, which are the major focus of this work. Structural studies are ongoing and will be reported in the future.

\subsection{Alloy Compositions and Composition Variations}

The average APT bulk and matrix solute compositions, in at.\%, are given in Tables 3 and 4 for the high and very high fluence conditions, respectively. The nominal bulk chemical values are given in parenthesis. Observed differences between the measured total and nominal values are largely expected. For example, the alloys that nominally contain $\approx 0.34 \%$ $\mathrm{Cu}$ have a lower average total APT content of $\approx 0.25 \%$. This value is consistent with previous observations of lower residual amounts in solution due to coarse scale preprecipitation during tempering and stress relief heat treatments, when the total $\mathrm{Cu}$ is beyond the solubility limit $[2,3,7,41]$. Likewise the Mn contents are lower than the nominal value since this element is partially sequestered in coarse $\mathrm{Mn}_{0.6} \mathrm{Fe}_{2.4} \mathrm{C}$ carbides. The quantitative differences between the nominal and measured bulk compositions are shown in Tables 3 and 4. 
These overall composition averages do not reflect the tip-to-tip variations in individual alloys, which are significant in some cases. For example, the standard error for the bulk Mn measurements of a given alloy is as large as $0.20 \%$. While seemingly an esoteric detail, we show below that the effects of the tip-to-tip composition variations in a given alloy are directly reflected in the precipitates, and are also consistent with observed alloy-to-alloy trends. A corollary is that it is important to use the actual local compositions in analyzing the APT precipitate data.

\subsection{High Fluence Condition}

Figure 2 shows atom maps for the highest Ni content, Cu-free steel (CM6) and the high $\mathrm{Ni}-\mathrm{Cu}$ content steel (LD) in the high fluence condition. The precipitates are numerous and well defined in the $\mathrm{Cu}$-bearing steel, but are not as easy to observe in the $\mathrm{Cu}$-free alloy; however, they are readily characterized by the cluster search algorithm. Table 5 summarizes the corresponding APT measurements. The left portion of the table gives the $\mathrm{Cu}-\mathrm{Mn}-\mathrm{Ni}-\mathrm{Si}$ compositions of the precipitates. The uncertainty is the standard error of the mean of the various measurements. The nominal precipitate Fe content is also shown to the right of the precipitate compositions. While we believe that the Fe is largely an APT artifact, it is included for those who might seek an alternative analysis. The right portion of Table 5 gives the average radius $(<\mathrm{r}\rangle)$, number density $(\mathrm{N})$ and mole fraction (f) of the MNS precipitates, as well as the standard error uncertainties in the measurements. The bar chart in Figure 3a summarizes the precipitate $\mathrm{f}$ and corresponding compositions. We will later show that the $\mathrm{f}$ directly scales with the individual tip solute contents. Thus Figure 3 a shows the mole fraction for individual tips with bulk compositions close to the average values of the alloy. The trends

for $\mathrm{Ni}$ content in $\mathrm{Cu}$-free steels, $\mathrm{Cu}$ content at medium $\mathrm{Ni}$ content, and $\mathrm{Cu}$ content at both the 
higher and the highest $\mathrm{Ni}$ content, are individually highlighted in the three sections. The corresponding $\langle\mathrm{r}\rangle$ and $\mathrm{N}$ are plotted in Figure $3 \mathrm{~b}$. The major observed trends are as follows: - The highest $\mathrm{Ni}$ content, $\mathrm{Cu}$-free steel has only a slightly larger $\mathrm{f}$ than the $\mathrm{Cu}$-free, medium Ni content alloy (Fig. 3a left). The Cu-Mn-Ni-Si contents of the precipitates, hence the total $\mathrm{f}$, increase with increasing $\mathrm{Cu}$ content in the medium Ni content steels (Fig. 3a center). The mole fraction of $\mathrm{Cu}$ in the precipitates increases from $\approx 0$, in the $\mathrm{Cu}$-free steel, to $0.15 \%$ in the highest $\mathrm{Cu}$ content steel. The average residual matrix $\mathrm{Cu}$ content is $0.06 \%$ in the $\mathrm{Cu}-$ bearing steels, indicating incomplete phase separation for this highly insoluble element.

- The increase in $\mathrm{f}$ with higher $\mathrm{Ni}$ content in the $0.21 \% \mathrm{Cu}$ steels is due to higher contents of $\mathrm{Ni}, \mathrm{Mn}$ and $\mathrm{Si}$ in the precipitates (Fig. 3a center and right). These results clearly demonstrate the thermodynamically driven synergisms between these elements, and the very important role played by $\mathrm{Ni}$. The corresponding effect of increasing $\mathrm{Ni}$ content on $\mathrm{f}$ in the $\mathrm{Cu}$-free and high $\mathrm{Cu}$ content steels is minimal in this case (Fig. 3a right) because $\mathrm{f}$ is so strongly dependent on $\mathrm{Cu}$.

- Both $\mathrm{N}$ and $\langle\mathrm{r}\rangle$ increase with $\mathrm{Cu}$ content (Fig. 3b). $\mathrm{N}$ also increases with $\mathrm{Ni}$ content, except in the $\mathrm{Cu}$-free steels. It should be noted that at these small $\mathrm{f}$ and $\langle\mathrm{r}\rangle$, the uncertainties in $\mathrm{N}$ are larger.

- The Mn-Ni-Si cluster compositions are generally similar in the medium and high Ni content steels (LG, LH, LI, LC, LD) with fractional averages and standard deviations of: $0.31 \pm 0.04$ $\mathrm{Mn}, 0.47 \pm 0.01 \mathrm{Ni}$ and $0.22 \pm 0.03 \mathrm{Si}$. The precipitate $\mathrm{Ni}$ fraction is higher and the $\mathrm{Si}$ is lower in the highest $\mathrm{Ni}$ content, $\mathrm{Cu}$-free steel (CM6) averaging $0.26 \mathrm{Mn}, 0.59 \mathrm{Ni}$ and $0.15 \mathrm{Si}$.

The most important observation for the high fluence condition is that $\mathrm{Cu}$ and $\mathrm{Ni}$ play a combined role in mediating $\mathrm{f}$, although $\mathrm{Cu}$ seems to have a stronger influence over the range 
of $\mathrm{Ni}$ compositions studied here. While the features in the Cu-bearing steels contain more than $75 \% \mathrm{Mn}-\mathrm{Ni}-\mathrm{Si}$, the total mole fraction of these elements roughly scales with the alloy $\mathrm{Cu}$ content. Thus higher $\mathrm{Cu}$ and $\mathrm{Ni}$ content lead to larger $\mathrm{f}$. These trends are consistent with previous lower flux-fluence SANS studies. However, the latter results will be described and analyzed in detail elsewhere.

The most plausible hypothesis for explaining these observations is that in the $\mathrm{Cu}$-free steels, the clusters are still predominantly matrix features. However, the presence of $\mathrm{Cu}$ catalyzes the formation of MNS precipitates. The $\mathrm{Cu}$ is highly supersaturated, thus precipitates quickly, while simultaneously being enriched in the other solutes. In the presence of $\mathrm{Cu}$, the difficult nucleation step for nearly pure $\mathrm{Mn}-\mathrm{Ni}$-Si phases is largely avoided by coprecipitation, and these solutes subsequently flow to the $\mathrm{Cu}$-catalyzed precipitates starting at relatively low fluence.

\subsection{Very High Fluence Condition}

Atom maps, for the same alloys as figure 2, but at the very high fluence condition are

shown in Figure 4. They clearly demonstrate the large changes that occur between the high versus very high fluence conditions. The changes in the precipitates are particularly notable in both the highest $\mathrm{Ni}$ content, $\mathrm{Cu}$-free (CM6) and the high $\mathrm{Ni}-\mathrm{Cu}$ content (LD) steels. The average precipitate parameters are summarized in Table 6 . These large changes are not surprising, since the fluence increased by a factor of $\approx 8$ between the high and very high fluence conditions. The major observations can be summarized as follows.

- There is a large increase in $\mathrm{f}$ between the high and very high fluence conditions. The histograms in Figure 5a, again for individual tips with bulk compositions close to the average values, show both the total $\mathrm{f}$ and precipitate composition for each alloy. These results 
demonstrate that the effect of $\mathrm{Cu}$ is not nearly as significant at very high versus high fluence, and that Ni plays a much stronger role than in the former case. At very high fluence, the MNS precipitates no longer need $\mathrm{Cu}$ to form. The precipitate $\mathrm{f}$ more than doubles in the $\mathrm{Cu}$ free steels with increasing bulk $\mathrm{Ni}$ from $\approx 0.7 \%$ to $1.6 \%$ (Fig. 5a left). In contrast, at medium Ni contents, increasing the bulk Cu only slightly increases f (Fig. 5a center); this increase is almost entirely caused by the higher amount of co-precipitate $\mathrm{Cu}$ itself. Notably, the highest $\approx 1.6 \% \mathrm{Ni}, \mathrm{Cu}-$ free steel (CM6) has a larger $\mathrm{f}$ than in the high $1.2 \% \mathrm{Ni}, 0.25 \% \mathrm{Cu}(\mathrm{LD})$ alloy (Fig. 5a right).

- The effects of $\mathrm{Cu}$ at both high and very high fluence are more clearly seen in the for $\mathrm{Mn}$ $\mathrm{Ni}$-Si only versus bulk $\mathrm{Cu}$ plots shown in Figure 5b. At high fluence (bottom - black dashed line), the Mn-Ni-Si $\mathrm{f}$ is minimal at $\approx 0.1 \%$ in the $\mathrm{Cu}$-free steels, but increases rapidly with larger alloy $\mathrm{Cu}$ contents. These results clearly show, as discussed previously, that $\mathrm{Cu}$ catalyzes the precipitation of the other Mn-Ni-Si solutes. In contrast at very high fluence (top), the Mn-Ni-Si f only increases slightly with higher bulk $\mathrm{Cu}$ content in the medium $\mathrm{Ni}$ content steels (purple dashed line). This is because the very high fluence is well beyond the range where $\mathrm{Cu}$ is needed to catalyze $\mathrm{Mn}-\mathrm{Ni}$-Si precipitation. Indeed, the small increase in the Mn-Ni-Si f is largely due to the fact that slightly higher Ni content happens to accompany the increase of $\mathrm{Cu}$ in this case. In contrast to the effect of $\mathrm{Cu}$, higher Ni contents cause a significant increase in Mn-Ni-Si $\mathrm{f}$ in the high Ni-Cu content alloy (LD - the red dotted line) and a much larger corresponding increase in the highest $\mathrm{Ni}$ content, $\mathrm{Cu}$-free steel (cyan data points).

- Figure $5 \mathrm{c}$ shows that $<\mathrm{r}>$ modestly increases with $\mathrm{Cu}$ in both medium and higher $\mathrm{Ni}$ content steels. In contrast, the corresponding $\mathrm{N}$ decreases with $\mathrm{Cu}$. In the case of the high $\mathrm{Ni}-\mathrm{Cu}$ 
content steel (LD), the very high fluence $\mathrm{N}$ is lower than for the high fluence condition, perhaps suggesting that the precipitates have begun to coarsen.

- Table 6 shows that at the very high fluence, the nominally $\mathrm{Cu}$-free steel precipitates contain $<0.01$ fraction of this element. In the medium $\mathrm{Ni}$ content steels, the precipitate $\mathrm{Cu}$ composition increases (LH, LI, LC) with this element up to $\approx 15 \%$ (LC). Figure 6 shows the relative precipitate $\mathrm{Mn}-\mathrm{Ni}$-Si compositions do not change much between high and very high fluences. For example, the average fractional compositional difference for the medium Ni content steels (LG, LH, LI, LC) is $0.02 \mathrm{Mn},-0.01 \mathrm{Ni}$ and $-0.01 \mathrm{Si}$ in going from high to very high fluence. Further, the $\mathrm{Mn}-\mathrm{Ni}-\mathrm{Si}$ precipitate compositions are generally similar in the medium Ni content steels at very high fluence (LG, LH, LI, LC) with fractional averages and standard deviations of: $0.35 \pm 0.03 \mathrm{Mn}, 0.44 \pm 0.02 \mathrm{Ni}$ and $0.20 \pm 0.02 \mathrm{Si}$. The precipitate $\mathrm{Mn}$ fraction is lower and $\mathrm{Ni}$ is higher in the high $\mathrm{Ni}-\mathrm{Cu}$ content steel (LD) at $0.25 \mathrm{Mn}, 0.52 \mathrm{Ni}$, and 0.23 Si. The corresponding composition of the highest $\mathrm{Ni}$ content, $\mathrm{Cu}$-free steel (CM6) is 0.36 Mn, $0.52 \mathrm{Ni}$ and $0.12 \mathrm{Si}$. Thus the precipitate compositions clearly reflect changes in the bulk alloy Ni and Si chemistry.

- $\mathrm{Cu}$ approaches full depletion at very high fluence, at a matrix level of $\approx 0.03 \%$, while there is still a modest fraction of the other elements left in solution. Figure 7 shows that $f$ approximately scales linearly with the total alloy $\mathrm{Cu}-\mathrm{Mn}-\mathrm{Ni}-\mathrm{Si}$ solute content, above an extrapolated minimum of $\approx 0.78$ at. $\%$ with a slope of $\approx 0.98$.

- Figure 8 illustrates the evolution of the morphology of precipitates with increasing fluence in the high $\mathrm{Ni}-\mathrm{Cu}$ content (LD) steel. Figure 8 a shows a cross section of a precipitate at high fluence, where there appears to be a $\mathrm{Mn}-\mathrm{Ni}-\mathrm{Si}$ shell surrounding a $\mathrm{Cu}$-rich core, consistent with previous experimental results and Lattice Monte Carlo simulations [15, 17, 18, 24]. 
Figure $8 \mathrm{~b}$ shows a precipitate at very high fluence, where there is a $\mathrm{Cu}$-rich core-shell structure, similar to that at high fluence, but this feature is associated with a nearly "pure" Mn-Ni-Si co-precipitate appendage. Such appendages have also been seen in irradiated surveillance steels [27].

In summary, the evolution between high and very high fluence is dominated by the continued flow of $\mathrm{Mn}-\mathrm{Ni}-\mathrm{Si}$ to the precipitates. In $\mathrm{Cu}$-free steels it is likely that the precipitates evolve from matrix feature solute-defect clusters initially formed in displacement cascades. At higher supersaturated $\mathrm{Cu}$ contents, $\mathrm{Cu}-\mathrm{Mn}-\mathrm{Ni}-\mathrm{Si}$ precipitates form rapidly and the $\mathrm{Mn}-\mathrm{Ni}$-Si co-precipitate appendages continue to grow after matrix $\mathrm{Cu}$ is depleted at higher fluence.

\subsection{Comparison of APT Precipitate Compositions with Potential Mn-Ni-Si Intermetallic}

\section{Phases}

The Mn-Ni-Si compositions of the precipitates in $\mathrm{Cu}$-free steels are similar to those in the co-precipitates formed in $\mathrm{Cu}$-bearing steels at very high fluence. These compositions can be compared to known intermetallic phases in the corresponding Mn-Ni-Si ternary and Mn-NiSi-Fe quaternary systems. As described in detail in a companion paper under submission, recent Calphad-Thermocalc computational modeling studies carried out at the University of Wisconsin (UW) have shown there are a variety of equilibrium Mn-Ni-Si intermetallic phases in the Fe-Mn-Ni-Si quaternary system at low RPV operating temperatures [42]. Briefly, these models were based on two different thermodynamic databases, including both "in-house" and commercial versions. The in-house database, assembled from literature, predicts the $\Gamma_{2}\left(\mathrm{Mn}_{2} \mathrm{Ni}_{3} \mathrm{Si}\right.$, T6) phase forms in all alloys at around $290^{\circ} \mathrm{C}$, while the commercial database predicts varying fractions of $\Gamma_{2}$ and $\mathrm{G}\left(\mathrm{Mn}_{6} \mathrm{Ni}_{16} \mathrm{Si}_{7}, \mathrm{~T} 3\right)$ phases, 
depending on the alloy Mn-Ni-Si composition, determined from the average APT values measured in this study.

Figure 9 shows the APT Mn-Ni-Si precipitate compositions (filled symbols) plotted on the Mn-Ni-Si ternary phase diagram for both the medium (circles) and high (squares) $\mathrm{Ni}$ content steels compared to the predicted average compositions for the commercial (open symbols) database. The commercial database predicts $100 \% \Gamma_{2}$ (T6) (open cyan square) for the highest Ni content, Cu-free (CM6) steel, which is very consistent with the APT data (filled cyan square). The commercial database predicts $\approx 73 \% \mathrm{G}(\mathrm{T} 3)$ and $\approx 27 \% \Gamma_{2}$ (T6) phases (open red square) for the high $\mathrm{Ni}-\mathrm{Cu}$ content (LD) steel, again very close to the measured value (filled red square). The medium Ni content alloys cluster around the Si-rich end of the $\Gamma_{2}$ phase field (filled circles), shown by the heavy green line at $\approx$ constant $\mathrm{Mn}$, while the commercial database predicts the presence of either $\mathrm{G}$ or $\Gamma_{2}$ phases (open circles).

The precipitates in the $\mathrm{Cu}$-free, medium Ni content steel (LG) have slightly less Mn and higher $\mathrm{Ni}$ than the other three medium $\mathrm{Ni}$ content steels that contain various amounts of $\mathrm{Cu}$. This might be interpreted to suggest that there is some effect of $\mathrm{Cu}$ on the dominantly $\mathrm{Mn}$ Ni-Si phases. However, these differences are actually highly consistent with variations in the bulk alloy $\mathrm{Ni}$ contents, that trade off with $\mathrm{Mn}$ in the precipitates, at approximately constant $\mathrm{Si}$. For example, the medium $\mathrm{Ni}$ content alloy with the greatest fraction of $\mathrm{Mn}$ in the precipitates (LH), which is furthest to the right of the $\Gamma_{2}$ line in the Mn-Ni-Si ternary (filled green circle), has a bulk $\mathrm{Mn} / \mathrm{Ni}$ of 1.6. On the other hand, the alloy furthest to the left of the $\Gamma_{2}$ line (LG, filled blue circle) has much less bulk Mn, with a bulk Mn/Ni of 1.2.

While the relatively good agreement between Thermocalc average composition predictions and the APT measurements is encouraging, there are also important differences 
that should be noted. In particular, the commercial database predicts the presence of multiple near-stoichiometric phases in some alloys. In contrast, the APT measurements show that there is a unimodal distribution of MNS precipitate compositions that decreases in extent with increasing precipitate size, converging to a relatively narrow range of $\mathrm{Mn}-\mathrm{Ni}-\mathrm{Si}$ at the largest $r_{p}$. The corresponding average compositions also appear to vary somewhat with $r_{p}$. The variation and spread in compositions with $r_{p}$ is an example of the additional insight available from APT measurements. However, this will require more detailed analysis, since, for example, nominal precipitate compositions and the spread in the measured compositions are affected by counting statistics, especially at small sizes. However, further discussion of this topic, and other opportunities, are beyond the scope of this paper.

As noted previously, the total APT local chemistries vary from tip to tip, allowing characterization of the effects of both smaller single-alloy and larger alloy-to-alloy changes in composition. Figure 10 shows the precipitate individual solute $\mathrm{f}$ as a function of local composition for $\mathrm{Cu}(\mathrm{a}), \mathrm{Ni}(\mathrm{b}), \mathrm{Mn}(\mathrm{c})$, and $\mathrm{Si}$ (d). The plots of $\mathrm{f}$ versus bulk solute for $\mathrm{Cu}$ and $\mathrm{Ni}$ form a tight band in both cases, with least square fit slopes of 0.95 and 0.89 , combined with thresholds of $\approx 0.01 \% \mathrm{Cu}$ and $\approx 0.05 \% \mathrm{Ni}$, respectively (Fig. $10 \mathrm{a}$ and b). In the case of $\mathrm{Mn}$, all the alloys fall into one tight band, except for the highest $\mathrm{Ni}$ content, $\mathrm{Cu}$ free (CM6) steel (Fig. 10c). The least square fit slope for the $0.8-1.2 \% \mathrm{Ni}$ steels is 0.36 with an intercept of $\mathrm{Mn} \approx-0.32 \%$. The corresponding least squares fit $\mathrm{Mn}$ slope for the highest $\mathrm{Ni}$ content, $\mathrm{Cu}$-free alloy is 0.80 with an intercept of $\mathrm{Mn} \approx 0.17 \%$. There are also two bands for the effect of Si on $\mathrm{f}$. The medium Ni content steels with varying Cu content (LG, LH, LI, LC) fall along a line with a least square fit slope of 0.80 and a threshold of $\mathrm{Si} \approx 0.07 \%$, while the least square fit slope is 0.63 with an intercept of $\mathrm{Si} \approx-0.14 \%$, for the highest $\mathrm{Ni}$ content, $\mathrm{Cu}$ - 
free and high $\mathrm{Ni}-\mathrm{Cu}$ content steels (CM6 and LD). Note the high Ni content fits are less reliable in the cases of $\mathrm{Mn}$ and $\mathrm{Si}$ since they are for only 1 and 2 alloys, respectively.

The very systematic behavior in precipitate $\mathrm{f}$ as a function of alloy composition will be used to develop physically based chemistry factors for advanced TTS embrittlement models. The most significant observation is that the precipitation behavior is consistent with two Mn$\mathrm{Ni}-\mathrm{Si}$ intermetallic phases at $\approx 550 \mathrm{~K}$ that are found in Thermocalc evaluations. Further at high fluence, $\mathrm{Ni}$ has a dominant role in precipitation, compared to $\mathrm{Mn}$ and $\mathrm{Si}$, in terms of both the strength of the effect itself, and the wider range of $\mathrm{Ni}$ content in the steels studied here. These results are also very consistent with previous observations on the effect of $\mathrm{Ni}$ and Mn on both the precipitates and hardening and also help clarify the role of Si [6-9, 13-23]. However, since developing robust physically based chemistry factor will require evaluation of a wider range of alloy compositions and irradiation conditions, this will not be discussed further in this paper.

\subsection{Consequences of Mn-Ni-Si Precipitates-Irradiation Hardening and Embrittlement}

While not the focus of the current study, it is useful to consider the consequences of the very large mole fraction of MNS precipitates to hardening and embrittlement, including at very high fluence. Figure 11 shows the predicted versus measured equivalent $\Delta \sigma_{\mathrm{y}}$ from the high flux, high and very high fluence BR2 and ATR-1 irradiations, along with lower flux and fluence IVAR data. The measured $\Delta \sigma_{\mathrm{y}}$ is based on direct tensile test data or 3.33 times the measured microhardness changes $\left(\Delta \mathrm{H}_{\mathrm{v}}\right)$. The high flux data are for a condition following a very mild post-irradiation anneal for $5 \mathrm{~h}$ at $350^{\circ} \mathrm{C}$ to remove thermally unstable features that result from the high dose rate effects, that are absent at low flux, while leaving the precipitates intact $[4,14,39]$. The average decrease in hardness for the very high fluence 
condition was $\approx 14 \mathrm{dph}\left(\mathrm{kg} / \mathrm{mm}^{2}\right)$ or a corresponding $\approx 47 \mathrm{MPa}$ decrease in the equivalent $\Delta \sigma_{\mathrm{y}}$

The precipitates act as dispersed barriers that pin dislocations. The individual precipitate contribution to the yield stress, absent all other contributions, $\sigma_{\mathrm{p}}$, is given by the relation

$$
\left.\sigma_{\mathrm{p}} \approx \mathrm{C} \alpha_{\mathrm{p}}\left(\mathrm{r}_{\mathrm{p}}\right) \mathrm{Gb} \sqrt{\mathrm{f}_{\mathrm{p}}} /<\mathrm{r}_{\mathrm{p}}\right\rangle
$$

Here $\mathrm{C} \approx 1.7$ is a constant that is determined by the Taylor factor (3.05) and obstacle planar spacing geometry, $\alpha_{p}\left(r_{p}\right)$ is a size dependent obstacle strength factor, $G$ is the shear modulus and $b$ is the Burgers vector. The $f_{p}$ and $\left\langle r_{p}\right\rangle$ are based on APT or SANS measurements. The $\alpha_{p}\left(r_{p}\right)$ is taken from a Russell-Brown (RB)-type model [43], empirically modified by fitting it to the estimated $\sigma_{\mathrm{p}}$ versus $\mathrm{f}_{\mathrm{p}}$ and $\mathrm{r}_{\mathrm{p}}$ data, resulting in a peak at $\left\langle\mathrm{r}_{\mathrm{p}}\right\rangle \approx$ $1.2 \mathrm{~nm}$ of $\sigma_{\mathrm{p}} / \sqrt{ } \mathrm{f}_{\mathrm{p}} \approx 5680 \mathrm{MPa}$ for the MNS phase dominated conditions. The corresponding fitted peaks for $\mathrm{Cu}$-enriched precipitates at lower fluence are $\sigma_{\mathrm{p}} / \sqrt{ } \mathrm{f}_{\mathrm{p}} \approx 4525$ and $4780 \mathrm{MPa}$ for medium and high $\mathrm{Ni}$ content alloys, respectively. The precipitate hardening contribution is combined with the unirradiated dispersed barrier strengthening contributions, $\sigma_{\mathrm{u}}$, like from $\mathrm{Mo}_{2} \mathrm{C}$ precipitates, using a $\alpha_{\mathrm{p}}(\mathrm{d})$ dependent strength superposition model [7]. Here $\sigma_{\mathrm{u}}$ is taken as $200 \mathrm{MPa}$. Further details are described elsewhere [2, 7].

There is good agreement between the predicted and measured values up to very large equivalent $\Delta \sigma_{\mathrm{y}} \approx 700 \mathrm{MPa}$. The fact that these steels studied are small, split-melt heats is sometimes raised as a concern. Thus it is important to emphasize that these 'model' steels have normal contents of all key alloying elements and embrittlement related solutes, and they were processed with conventional heat treatment schedules. Their microstructures and irradiation responses are virtually identical to those observed in actual service pressure vessel 
alloys, with the exception that they are perhaps cleaner, and do not contain coarse scale impurity phases like $\mathrm{MnS}$ and $\mathrm{CuS}$.

Thus the $\Delta \sigma_{\mathrm{y}}$ can be used to estimate the TTS. Of course such property-property transfers carry some degree of uncertainty. However, they are based on a solid physical foundation, derived from finite element simulations of the various tests using the same true stress-strain constitutive law $[40,44]$. The corresponding estimated TTS in the toughness-temperature master curve $100 \mathrm{MPa} \sqrt{\mathrm{m}}$ reference temperature $\left(\mathrm{TTS}=\Delta \mathrm{T}_{\mathrm{o}}\right.$ ) at the very high fluence and $\Delta \sigma_{\mathrm{y}}$ condition is $\Delta \mathrm{T}_{\mathrm{o}} \approx 0.7 \Delta \sigma_{\mathrm{y}}$, or up to a remarkable $\approx 500^{\circ} \mathrm{C}$ [45]. For any reasonable flux adjusted effective fluence, the power reactor end of life TTS would be lower. Based on our best estimate interpolation, this TTS would be $\approx 210^{\circ} \mathrm{C}$ in the highest $\mathrm{Ni}$ content, $\mathrm{Cu}$-free steel (CM6) and $\approx 330^{\circ} \mathrm{C}$ in the high $\mathrm{Ni}-\mathrm{Cu}$ content steel (LD). However, we acknowledge that these estimates are uncertain and more research is needed to quantify the flux effect.

\section{Summary, Conclusions and Future Work}

Large mole fractions of Mn-Ni-Si dominated LBP precipitates form in both $\mathrm{Cu}$-free and Cu-bearing RPV steels at very high fluence. Consistent with longstanding predictions:

- In Cu-free steels the MNS precipitates likely evolve from defect-solute cluster matrix feature precursors that constitute only a small $\mathrm{f} \approx 0.1 \%$ at high fluence, but subsequently grow to much larger mole fractions at very high fluence.

- Cu catalyzes the initial formation of MNS precipitates. This is especially the case of rapidly forming precipitates at the high supersaturations of $\mathrm{Cu}$. Thus at the high flux-high fluence condition in this study, $\mathrm{Cu}$ and $\mathrm{Ni}$ are the primary compositional variables leading to large mole fractions of MNS. 
- However, since the precipitates are dominated by $\mathrm{Mn}-\mathrm{Ni}-\mathrm{Si}, \mathrm{Cu}$ has a much weaker effect at very high fluence, while Ni has the major influence on the $\mathrm{f}$.

- The precipitate $\mathrm{Si}$ in the highest $\mathrm{Ni}$ content, $\mathrm{Cu}$-free (CM6) steel is significantly lower, in part due to the corresponding lower bulk Si content in this alloy. However, lower Si trades off with higher $\mathrm{Mn}$ and $\mathrm{Ni}$ so the net $\mathrm{f}$ is relatively unaffected. Indeed, we will show elsewhere that there is a close correlation between a simple alloy chemistry factor $2 \mathrm{Ni}+\mathrm{Cu}$ and $\mathrm{f}$.

- The increase in precipitate mole fractions leads to correspondingly large increases in hardness; hence, also leads to severe embrittlement.

- The experimental results in this study compare favorably to calculations using CalphadThermocalc models and associated databases. The precipitates in the high $\mathrm{Ni}-\mathrm{Cu}$ content (LD) steel are close to $\mathrm{G}$ phase $\left(\mathrm{Mn}_{6} \mathrm{Ni}_{16} \mathrm{Si}_{7}\right)$, while they are close to the $\Gamma_{2}$ phase $\left(\mathrm{Mn}_{2} \mathrm{Ni}_{3} \mathrm{Si}\right)$ in the highest Ni content, $\mathrm{Cu}$-free (CM6) steel. The precipitate compositions in the other medium Ni content alloys were in reasonably good agreement with the Si-rich end of the $\Gamma_{2}$ phase field, although the Thermocalc predictions vary somewhat from these compositions in this case.

There is a significant amount of additional detailed information and that can be derived from the APT studies. Further, future work will include other alloys and irradiation-annealing conditions. The resulting APT database will also be complimented by measurements based on other techniques, such as transmission electron microscopy and X-ray diffraction, as well as neutron and X-ray scattering. These tools will be important to characterizing the actual structure of the MNS phases that appear to be diffracting in preliminary TEM studies. Coupled with mechanical property data, this experimental knowledge base will be used to 
develop new physically based models of high fluence-low flux irradiation hardening and embrittlement.

\section{Acknowledgements}

A portion of this research, both the ATR irradiation and FIB/APT at the Center for Advanced Energy Studies - Microscopy and Characterization Suite (CAES-MaCS), was supported by the Advanced Test Reactor National Scientific User Facility through the U.S. Department of Energy, Office of Nuclear Energy under DOE Idaho Operations Office Contract DE-AC07-051D14517. Special thanks go to Colin Knight at the Idaho National Laboratory for assisting in the complex steps needed to gain access to the irradiated ATR specimens. The much earlier piggyback BR2 irradiations were sponsored by Jean Claude Van Duysen of Electricite de France and carried out under the supervision of Lorenzo Malerbra at SCK Belgium. We also thank Peter Hosemann at UC Berkeley for access to their FIB to prepare APT samples from the high fluence (BR2) condition. The MRL Shared Experimental Facilities were used for performing APT on the high fluence (BR2) samples and are supported by the MRSEC Program of the NSF under Award No. DMR 1121053 as a member of the NSF-funded Materials Research Facilities Network (www.mrfn.org). The bulk of this research was supported by the DOE Office of Nuclear Energy's Nuclear Energy University Program, as part of the LRW Sustainability Task. 


\section{References}

[1] Nuclear Power Plant Life Management and Longer-term Operation, OECD Nuclear Energy Agency (2006) NEA No. 6105.

[2] Eason E, Odette GR, Nanstad R, Yamamoto T. ORNL/TM-2006/530 2007.

[3] Eason E, Odette GR, Nanstad R, Yamamoto T. J Nucl Mater 2013;433:240-54.

[4] Odette GR, Nanstad R. JOM 2009;61(7): 17-23.

[5] Odette GR. Scripta Metall 1983;17(10):1183-8.

[6] Odette GR. Mater Res Soc Symp Proc 1995;373:137-48.

[7] Odette GR, Lucas GE. Radiat Effects Defects Solids 1998;144:189-231.

[8] Odette GR, Wirth BD. J Nucl Mater 1997;251:157-71.

[9] Wirth BD. On the Character of the Nano-Scale Features in Reactor Pressure Vessel Steels, $\mathrm{PhD}$ Thesis, University of California Santa Barbara 1998.

[10] Odette GR, Lucas GE. Proceedings of the 2nd International Symposium on Environmental Degradation of Materials for Nuclear Power Systems-Water Reactors, ANS 1986;295.

[11] Lucas GE, Odette GR, Chen H. Proceedings of the 2nd International Symposium on Environmental Degradation of Materials for Nuclear Power Systems-Water Reactors, ANS1986;345.

[12] Odette GR, Lucas GE. 14th International Symposium on the Effects of Irradiation on Materials, ASTM-STP-1046, ASTM1989;323.

[13] Odette GR. Irradiation Effects on Pressure Vessel Steels, IAEA IRRWG-LMNPP-98-3, IAEA $1998 ; 438$.

[14] Mader E. Kinetics of irradiation embrittlement and the post-irradiation annealing of nuclear reactor pressure vessel steels, PhD Thesis, University of California Santa Barbara 1995.

[15] Liu CL, Odette GR, Wirth BD, Lucas GE. Mater Sci Eng A 1997;238(1):202-9.

[16] Odette GR, Liu CL, Wirth BD. Mater Res Soc Symp Proc 1997;439:457-69.

[17] Miller MK, Russell KF. J Nucl Mater 2007;371:145-60.

[18] Miller MK, Russell KF, Sokolov MA, Nanstad RK. J Nucl Mater 2007;361:248-61. 
[19] Hyde JM, Ellis D, English CA, Williams TJ. 20th ASTM International Symposium on Effects of Radiation on Nuclear Materials, ASTM STP 14052001;262.

[20] Hyde JM, Sha G, Marquis EA, Morley A, Wilford KB, Williams TJ. Ultramiscroscopy 2011;111:664-71.

[21] Styman PD, Hyde JM, Wilford K, Smith GD. Ultramicroscopy 2013;132:258-64.

[22] Carter RG, Soneda N, Dohi K, Hyde JM, English CA, Server WL. J Nucl Mater 2001;298:211-24.

[23] Buswell JT, Phythian WJ, McElroy RJ, Dumbill S, Ray PHN, Mace J, Sinclair RN. J Nucl Mater 1995;225:196-214.

[24] Wirth BD, Odette GR, Pavinich W, Lucas GE, Spoone S. 18th International Symposium on the Effects of Radiation on Materials, ASTM STP-1325 1999;102.

[25] Odette GR, Yamamoto T, Wirth BD. Proceeding of the Second International Conference on Multiscale Modeling2004;105.

[26] Miller MK, Chernobaeva AA, Shtrombakh YI, Russell KF, Nanstad RK, Erak DY, Zabusov OO. J Nucl Mater 2009;385:615-22.

[27] Miller MK, Powers KA, Nanstad RK, Efsing P. J Nucl Mater 2013;437:107-15.

[28] Odette GR, Cowan C. CD Proceedings of the 10th International Symposium on Environmental Degradation of Materials in Light Water Reactors, National Association of Corrosion Engineers 2001.

[29] Odette GR, Yamamoto T, Klingensmith D. Philos Mag 2005;85(4-7):779-97.

[30] Gault B, Moody M, Cairney J, Ringer S. Atom Probe Microscopy. New York: Springer Series in Materials Science 2012.

[31] Miller MK, Atom Probe Tomography: analysis at the atomic level. New York: Kluwer Academics/Plenum; 2000.

[32] Stephenson LT, Moody MP, Liddicoat PV, Ringer SP. Microsc Microanal 2007;13:448-63.

[33] Marquis EA, Hyde JM. Mater Sci Eng R 2010;69:37-62.

[34] Marquis EA, Vurpillot F. Microsc Microanal 2008;14:561-70. 
[35] Cunningham NJ, Study of the Structure, Composition, and Stability of Y-Ti-O nm-Scale Features in Nano-Structured Ferritic Alloys, PhD Thesis, University of California Santa Barbara 2012.

[36] Ke H. Personal Communication 2013.

[37] Miller MK, Russell KF, Pareige P, Starink MJ, Thomson RC. Mater Sci EngA 1998;250:4954.

[38] Perez M, Perrard F, Massardier V, Kleber X, Deschamps A, De Monestrol H, Pareige P, Covarel G. Philos Mag 2005;85(20):2197-2210.

[39] Odette GR, Mader EV, Lucas GE, Phythian WJ, English CA.16 $6^{\text {th }}$ International Symposium on the Effects of Radiation on materials, ASTM-STP 1175 1993;373.

[40] He MY, Odette GR, Yamamoto T, Klingensmith D. J Nucl Mater 2007;367-370:556-60.

[41] Odette GR, Lucas GE.Proceedings of the 3rd International Symposium on Environmental Degradation of Materials for Nuclear Power Systems-Water Reactors, TMS 1987;95.

[42] Xiong W, Ke H, Wells P, Barnard L, Krishnamurthy R, Odette GR, Morgan D. "Thermodynamic models of low temperature $\mathrm{Mn}-\mathrm{Ni}$-Si precipitation in reactor pressure vessel steels," MRS Comm 2014; accepted.

[43] Russell KC, Brown LM. Acta Met 1972;20:969-74.

[44] Odette GR, He MY, Yamamoto T. J Nucl Mater 2007;367:561-567.

[45] Odette GR, He MY. J Nucl Mater 2000;283-287:120-127. 


\section{Figure Captions}

Figure 1. (a) $\Delta \sigma_{\mathrm{y}}$ and (b) $\mathrm{f}_{\mathrm{v}}$ in a high $\mathrm{Cu}$, medium Ni content steel (LC) for various irradiation conditions as a function of square root of the fluence showing a delay in both hardening and precipitate $\mathrm{f}_{\mathrm{v}}$ with increasing flux.

Figure 2. Atom Maps for the highest $\mathrm{Ni}$ content, $\mathrm{Cu}$-free (top), and high $\mathrm{Ni}-\mathrm{Cu}$ content (bottom) alloys irradiated to high fluence.

Figure 3. (a) The high fluence precipitate $\mathrm{f}$ for the individual solute elements for a single run of all 6 alloys, and (b) $<\mathrm{r}>$ and $\mathrm{N}$ plotted as a function of $\mathrm{Cu}$ for both the medium $\mathrm{Ni}$ and high $\mathrm{Ni}$ content steels. Note that in some cases, the error bars were smaller than the size of the symbols.

Figure 4. Atom Maps for the highest $\mathrm{Ni}$ content, $\mathrm{Cu}$-free (top) and high Ni-Cu content (bottom) alloys irradiated to very high fluence.

Figure 5. The $\mathrm{Cu}$ dependence of: (a) f showing the constituent elements for individual runs of each alloy, (b) Mn-Ni-Si f at both high and very high fluence, and (c) $<\mathrm{r}>$ and $\mathrm{N}$ at very high fluence. Note that in some cases, the error bars were smaller than the size of the symbols.

Figure 6. The average precipitate $\mathrm{Mn}-\mathrm{Ni}-\mathrm{Si}$ compositions at high and very high fluence.

Figure 7. The precipitate $\mathrm{f}$ as a function of the bulk $\mathrm{Cu}+\mathrm{Ni}+\mathrm{Mn}+\mathrm{Si}$ alloy content for individual tips at very high fluence.

Figure 8. APT maps of typical precipitates in the high $\mathrm{Ni}-\mathrm{Cu}$ content steel (LD): (a) at high fluence, and (b) at very high fluence. 
Figure 9. A Gibbs triangle showing APT Mn-Ni-Si precipitate compositions (filled symbols) at very high fluence compared to UW Thermocalc predictions for a commercial database (open symbols) [42].

Figure 10. The precipitate f for individual elements as a function of their corresponding bulk solute compositions.

Figure 11. Predicted vs. measured yield stress increases from the various previous UCSB irradiations, including the two conditions presented in this study. 

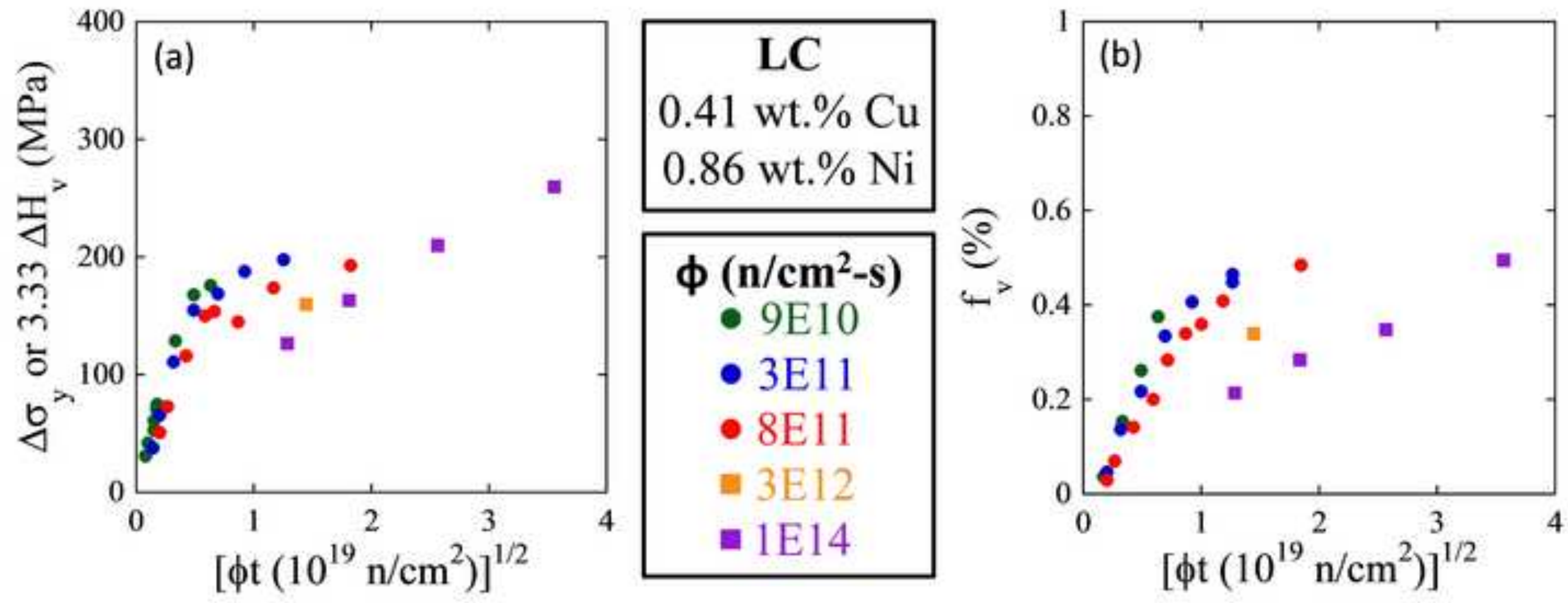


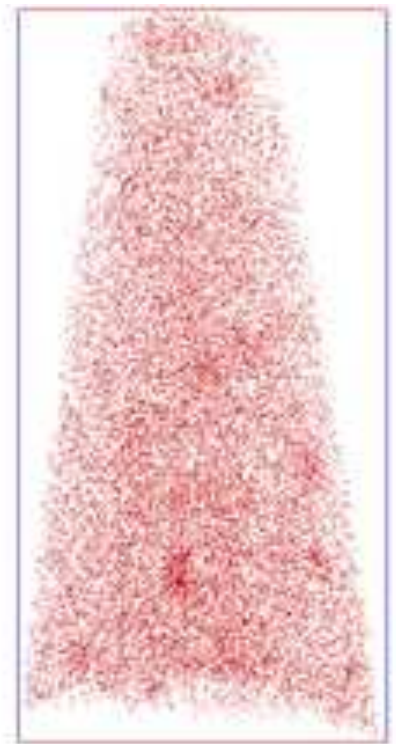

$\mathrm{Ni}$

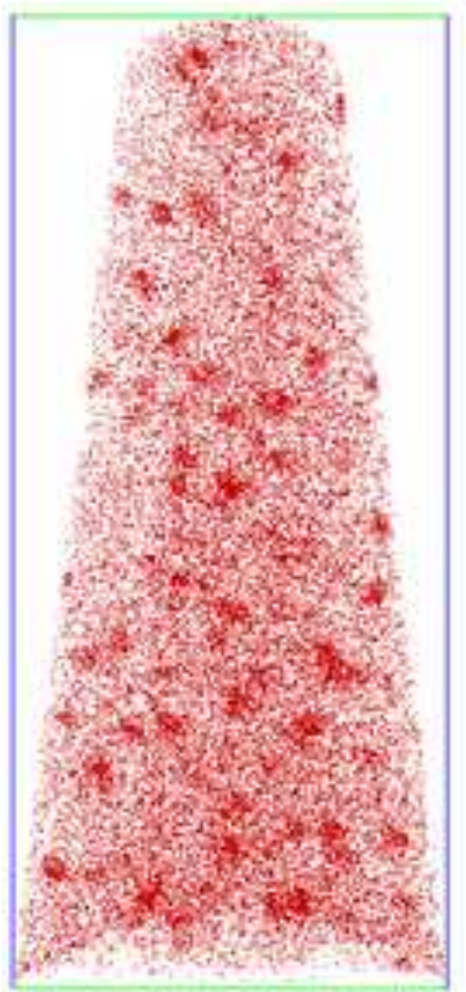

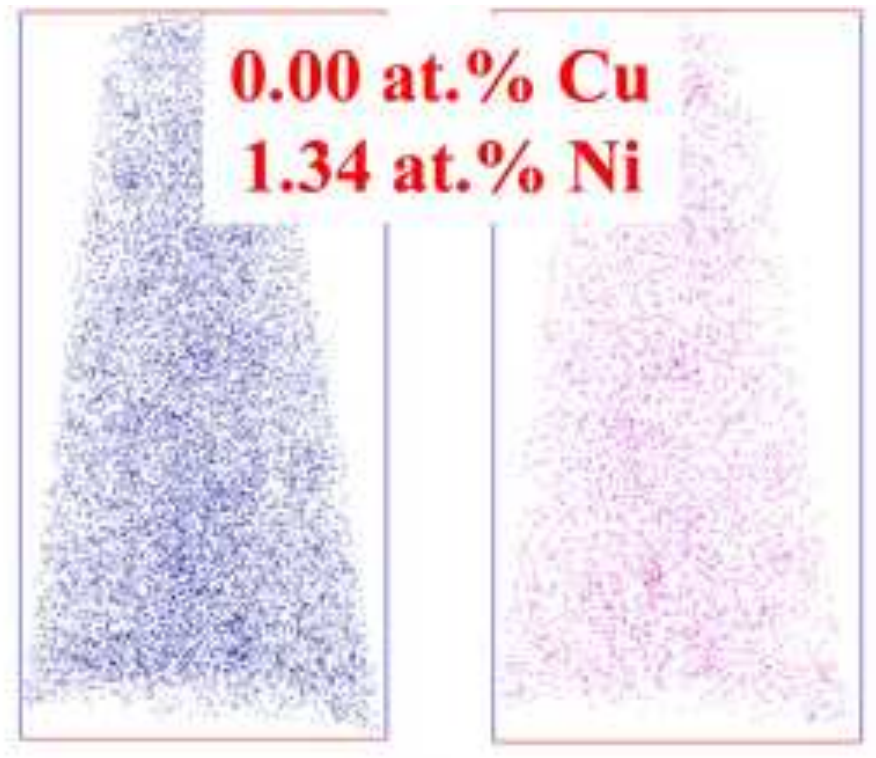

\section{$\mathrm{Mn} \underline{20 \mathrm{~nm}} \mathrm{Si}$}

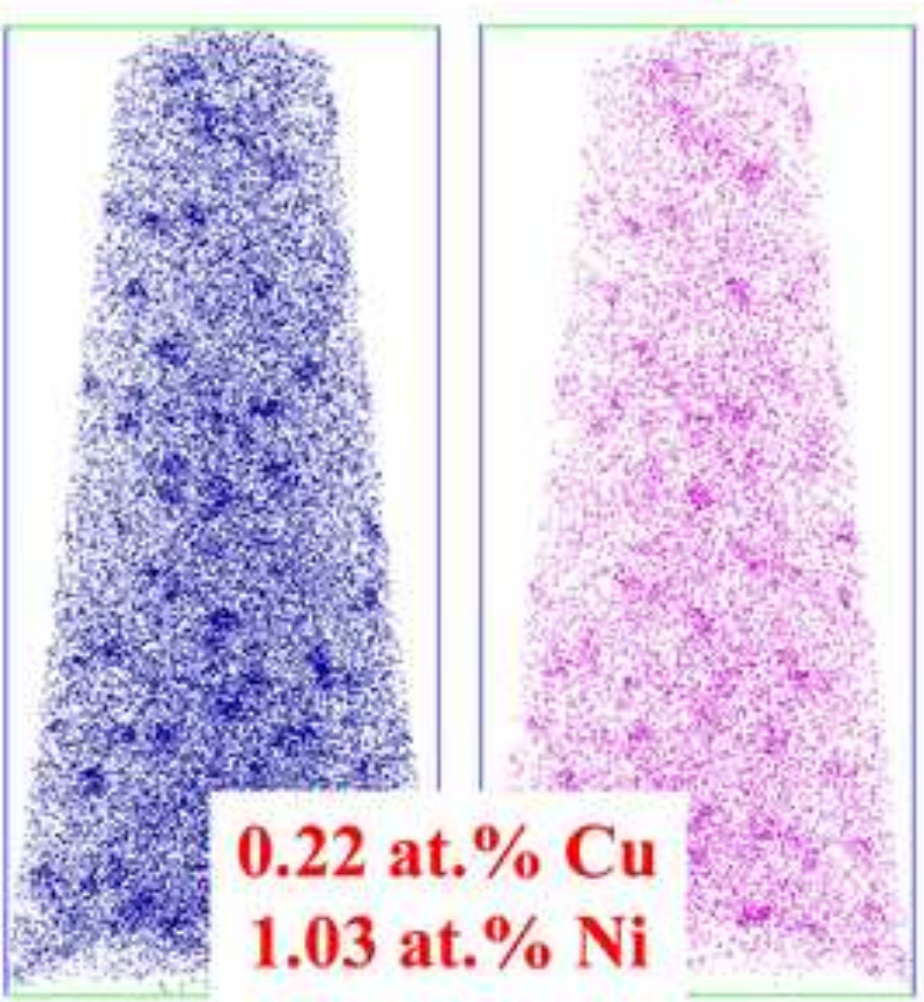

$\% \mathrm{Ni}$

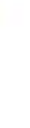

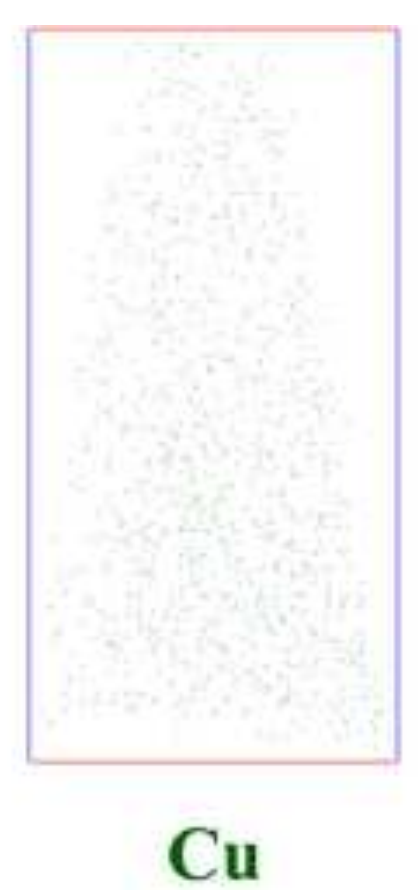

$\mathrm{Cu}$

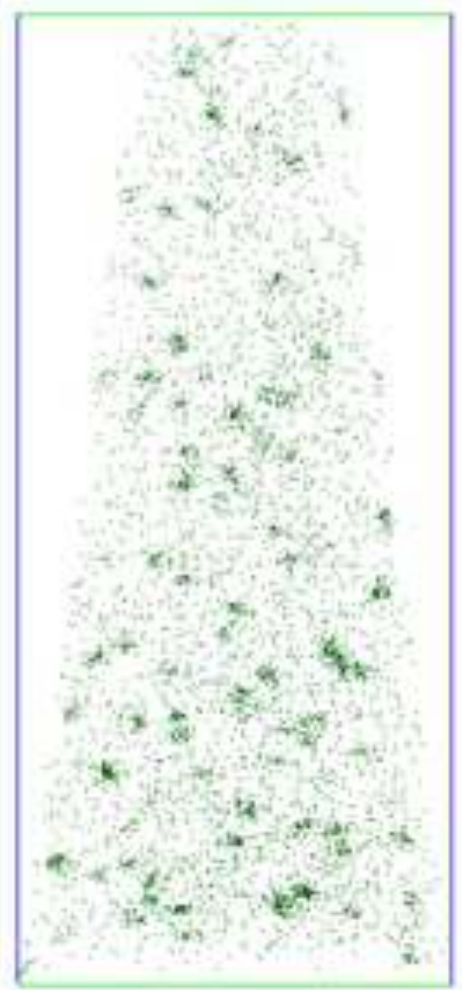




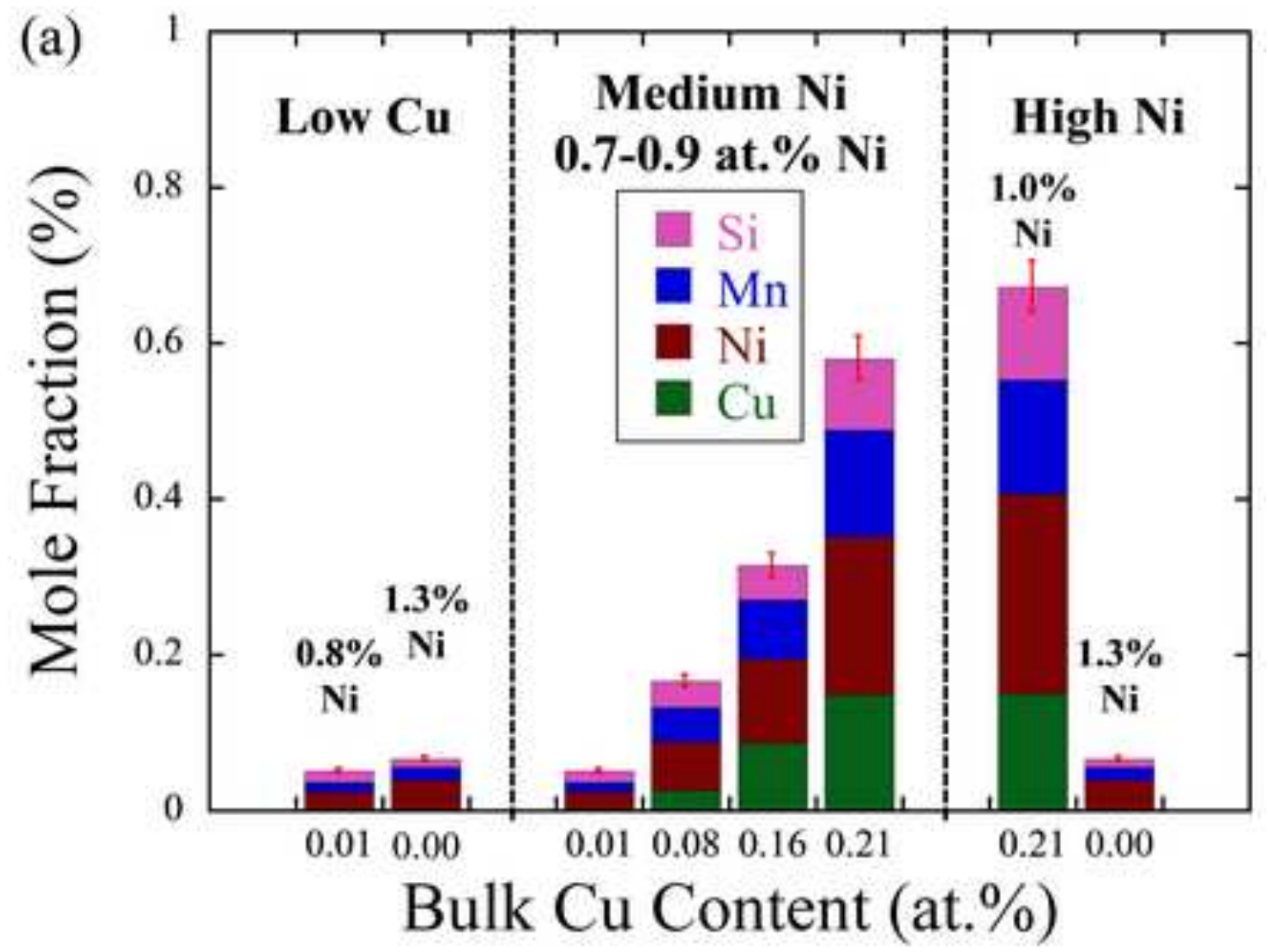

(b)
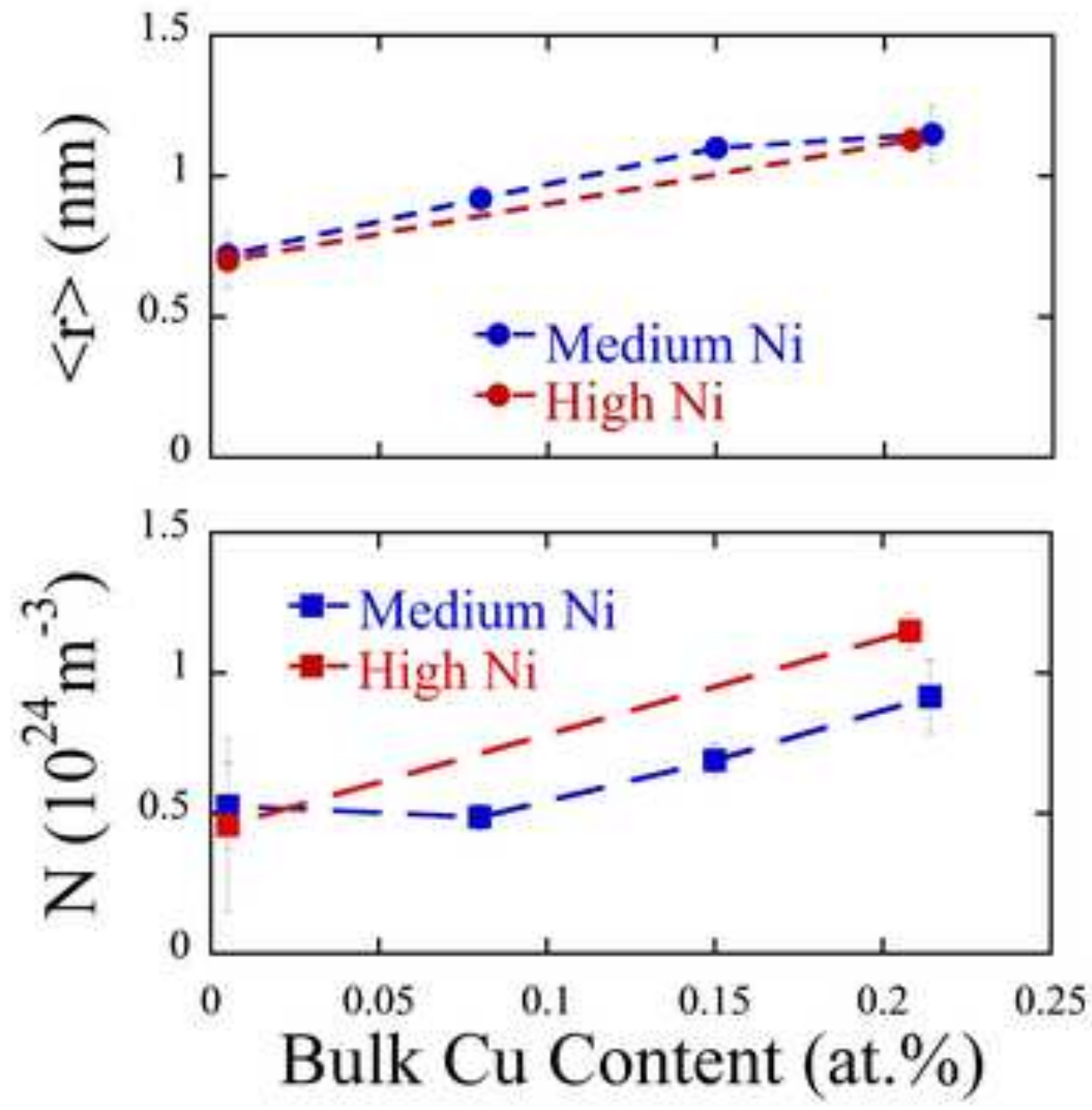


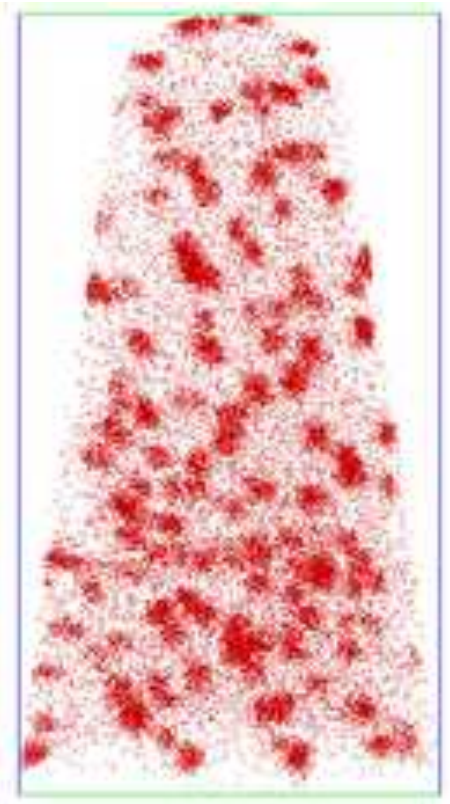

Ni

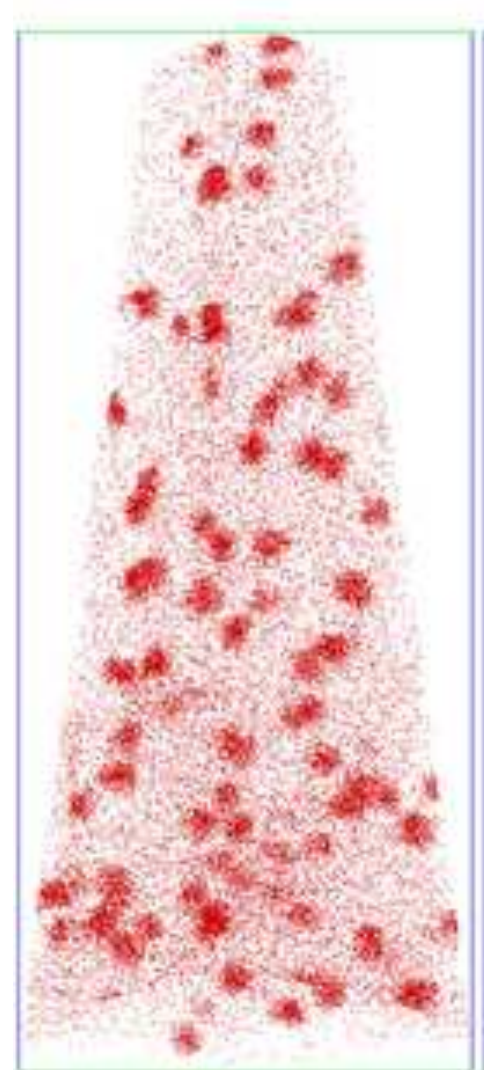

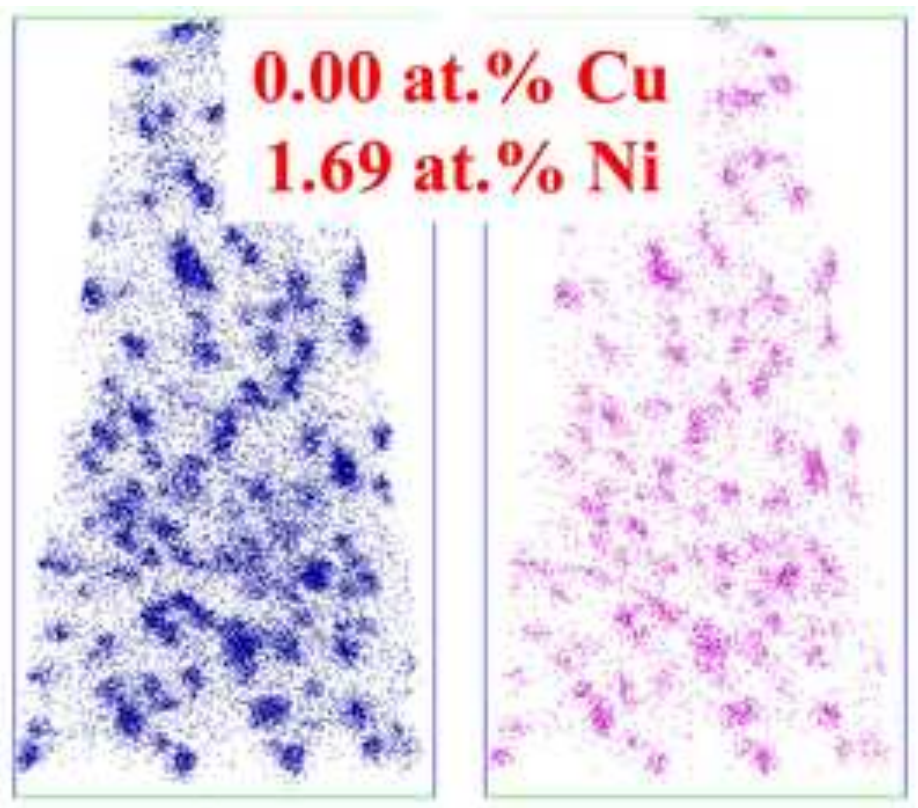

Mn $\underline{20 n m ~ S i ~}$

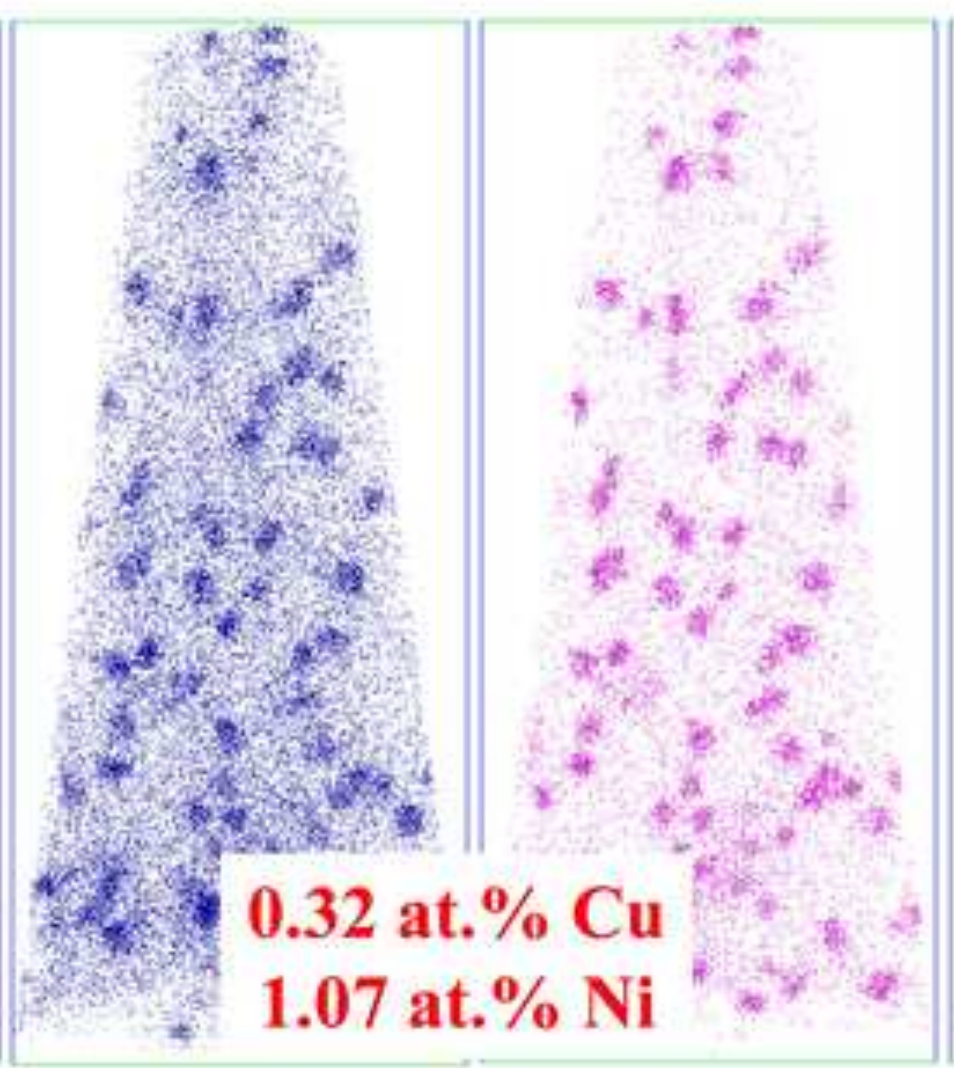

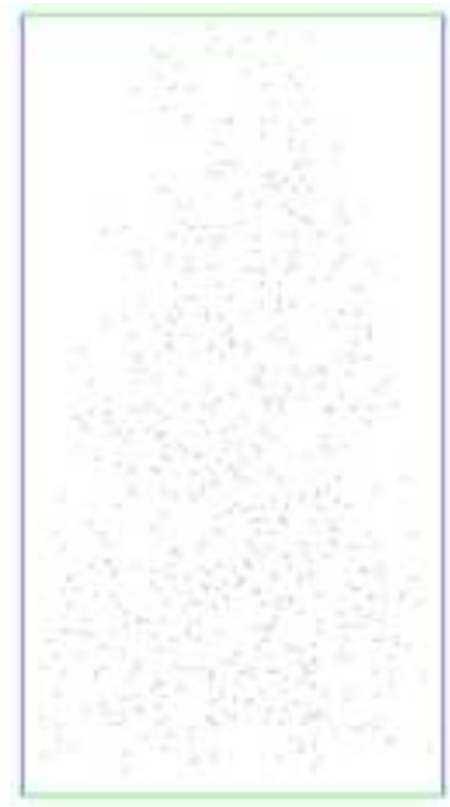

$\mathrm{Cu}$

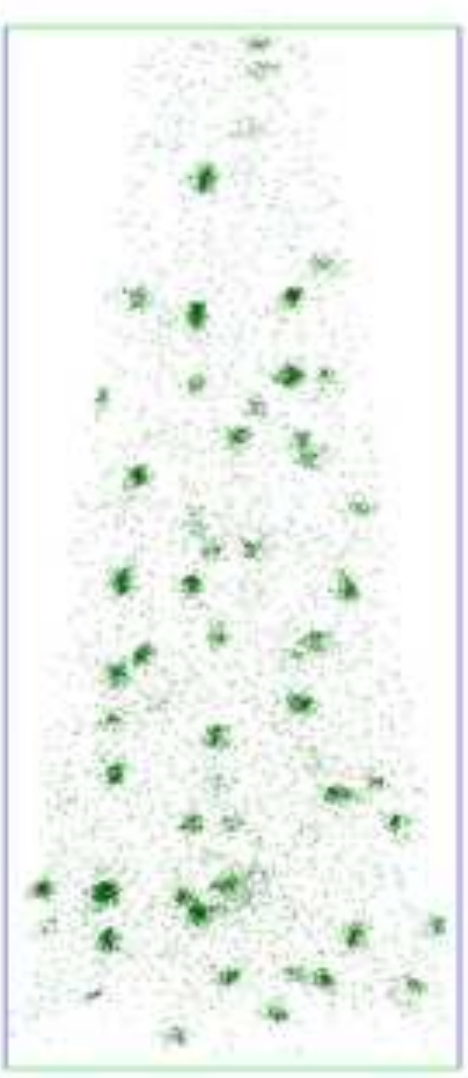

\section{Figure 4}

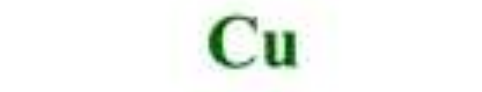


(a)

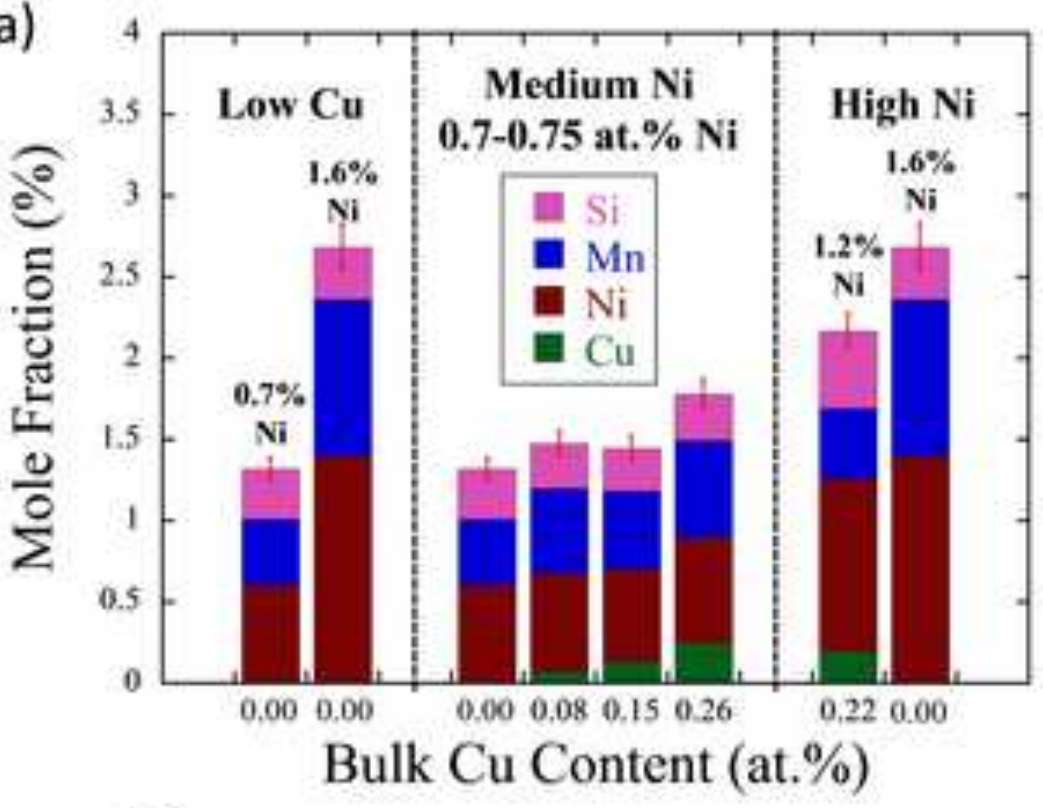

(b)

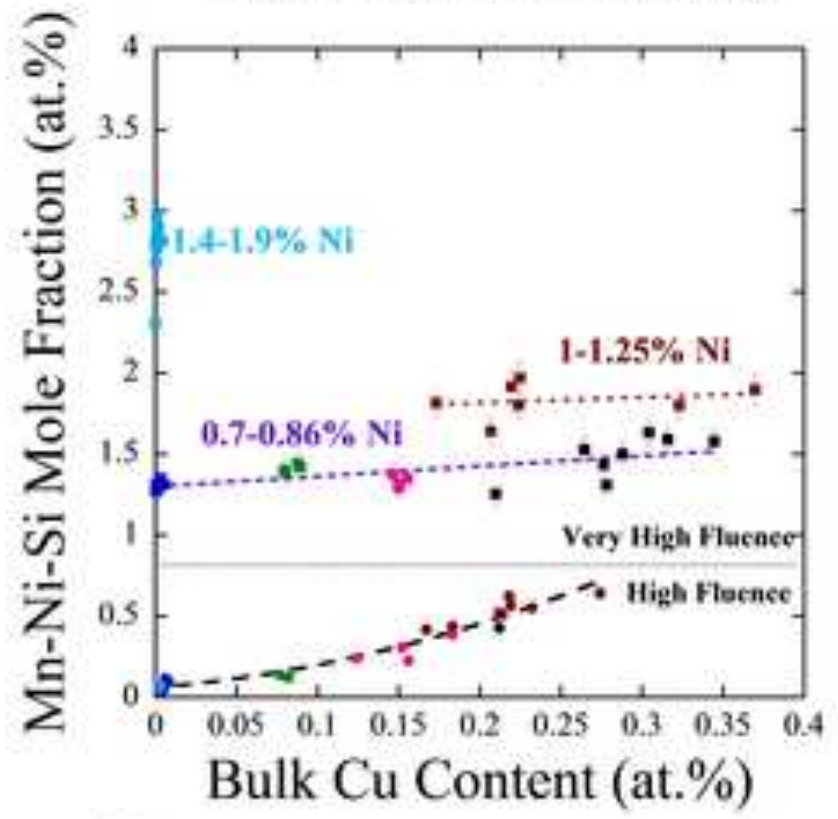

(c)
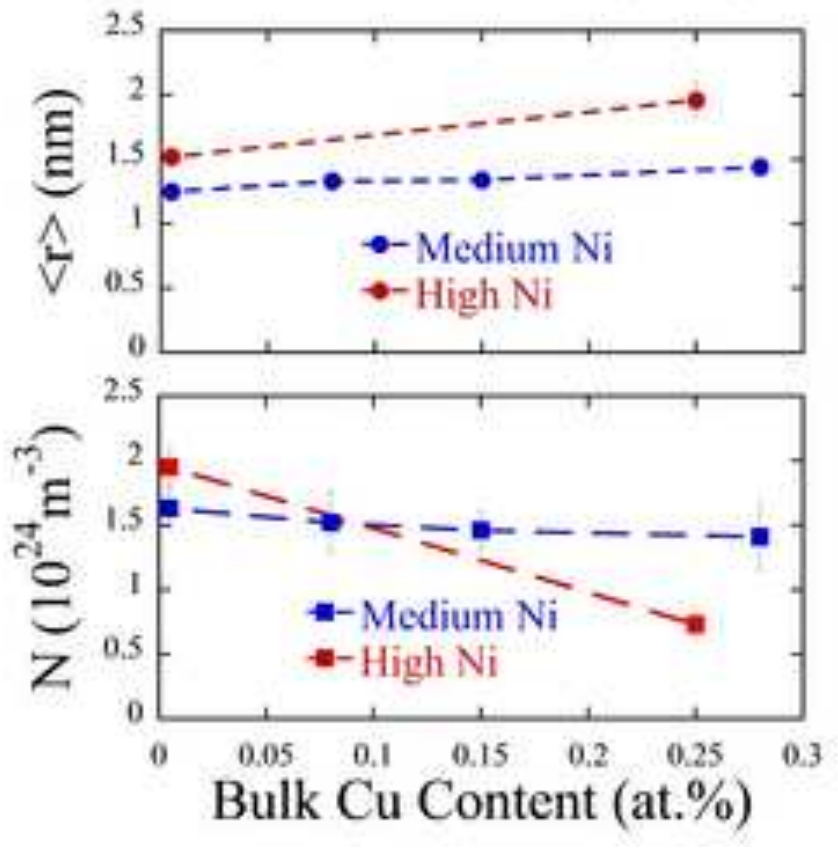


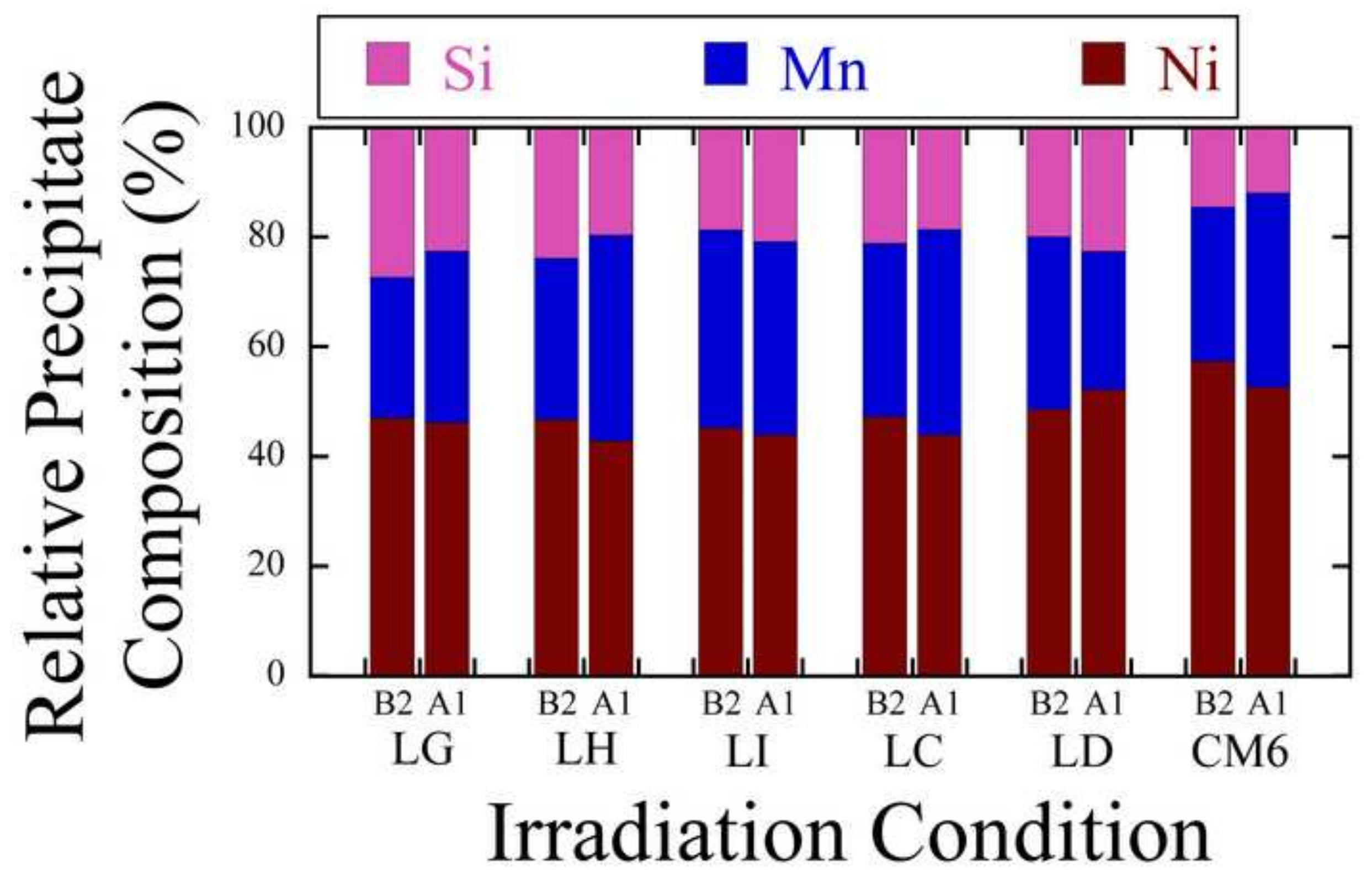




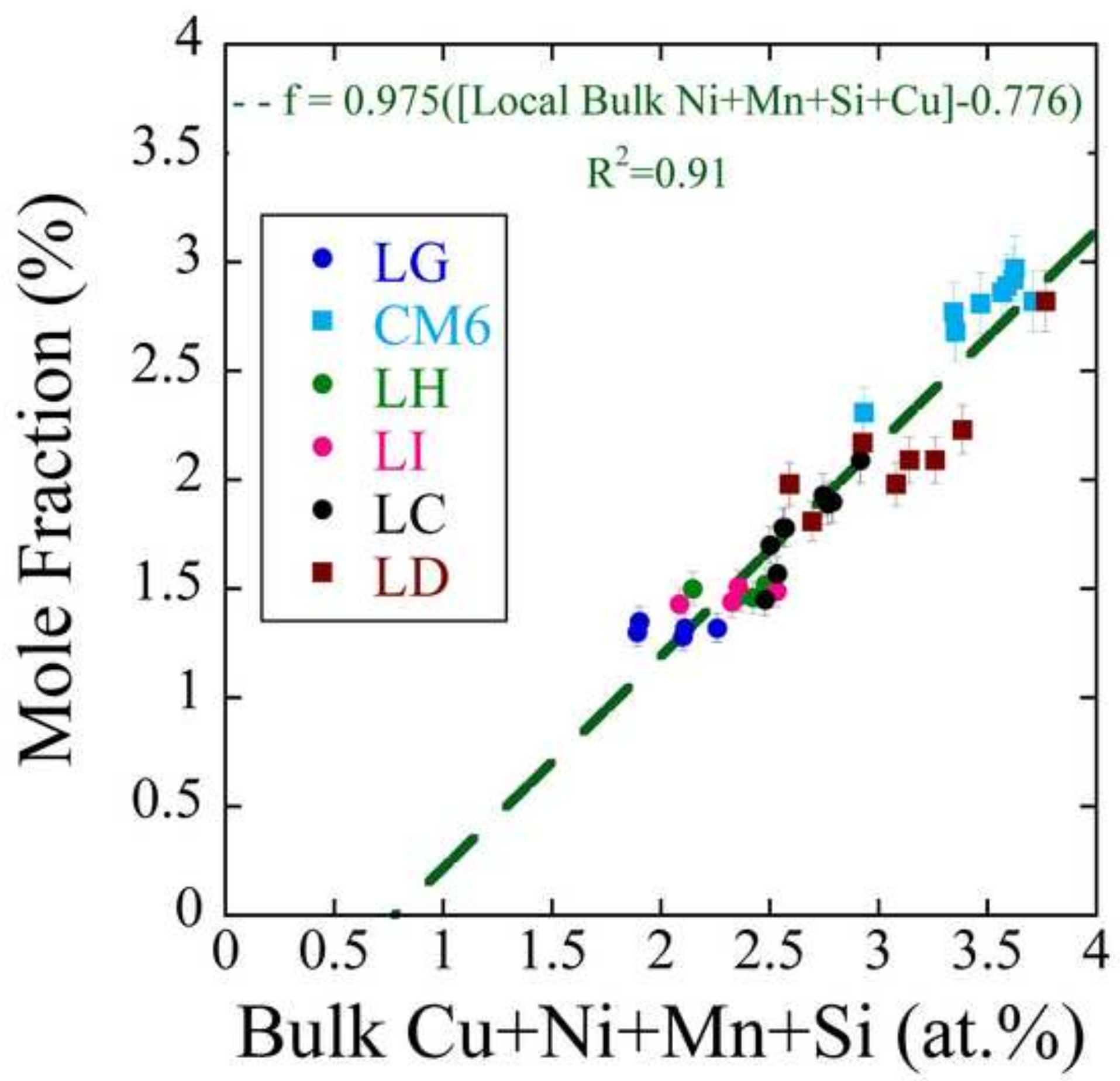


(a)
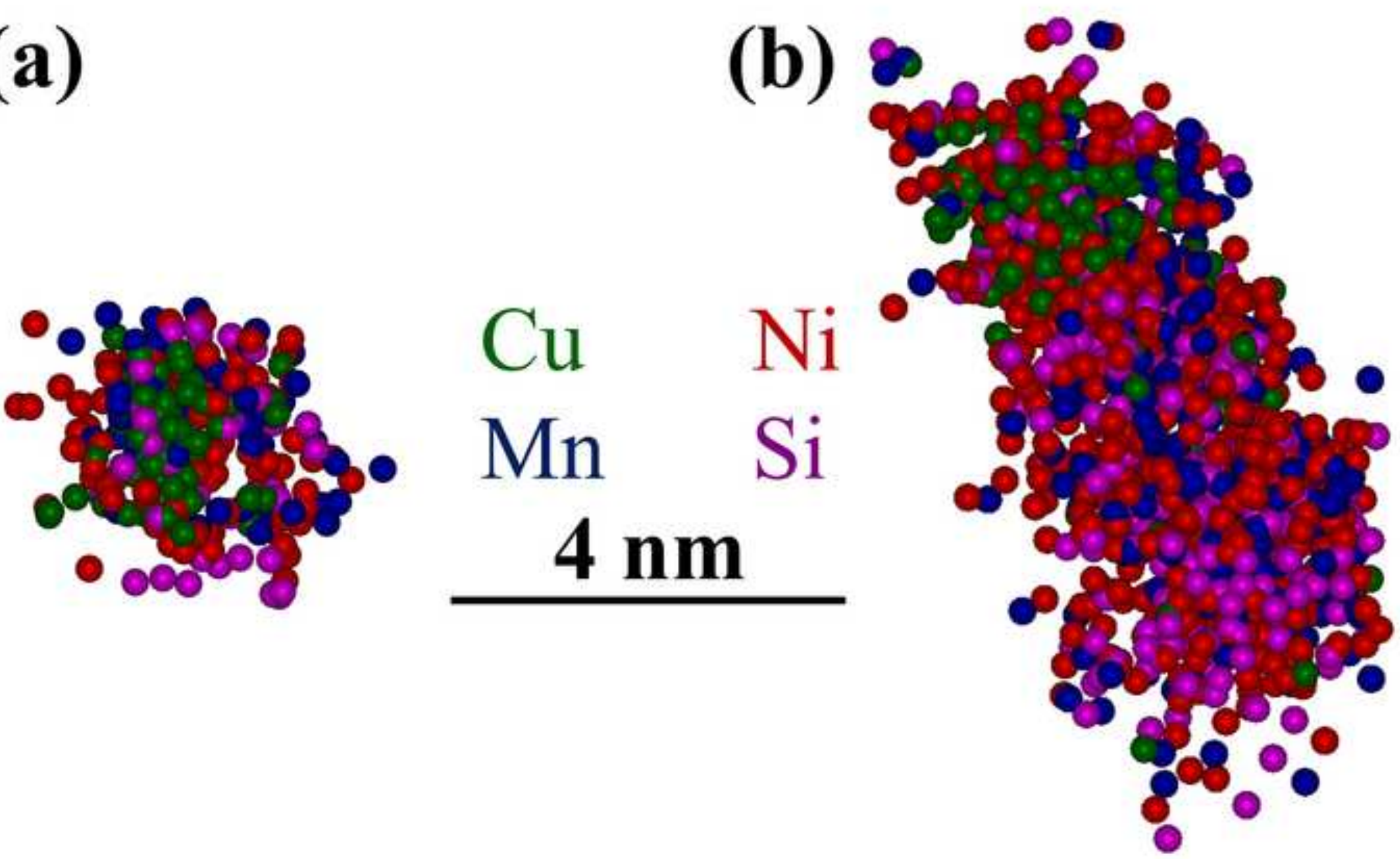
Medium Ni Alloys

- LG OLI

- LH OLC
* Open symbols are predictions from commercial database

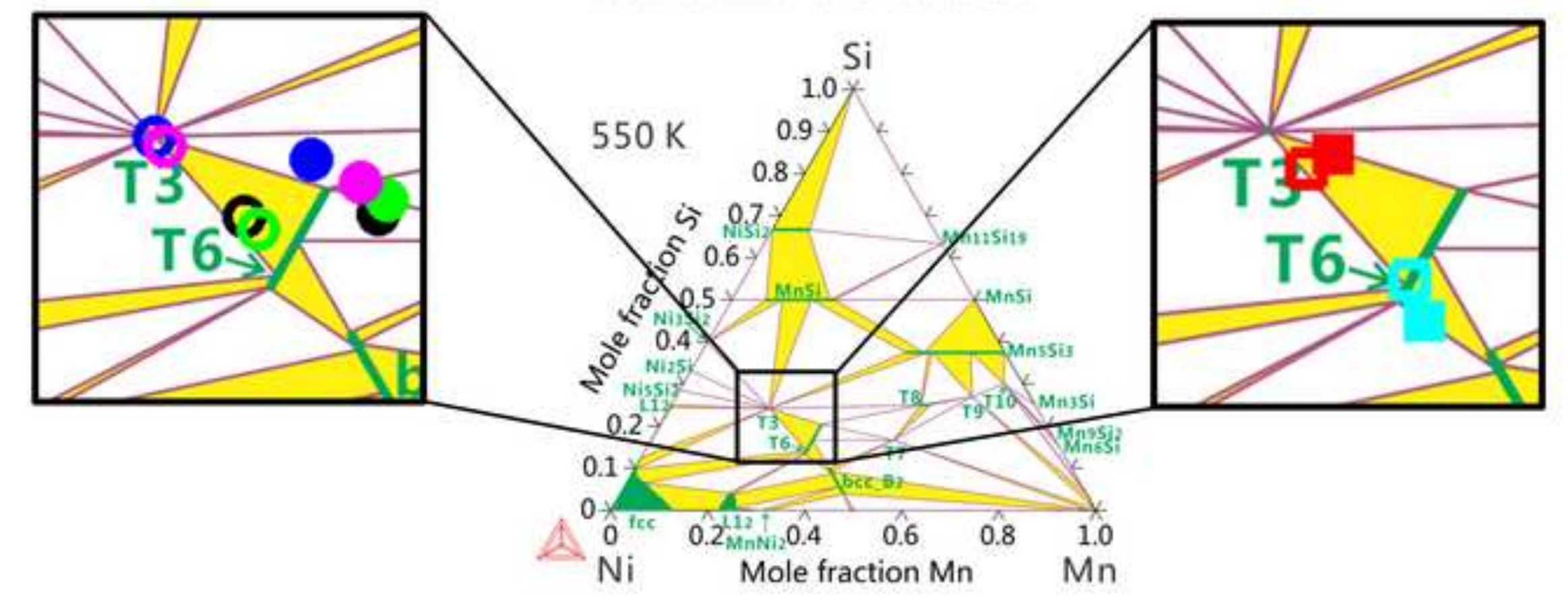

High Ni Alloys

an6

ㅁD 


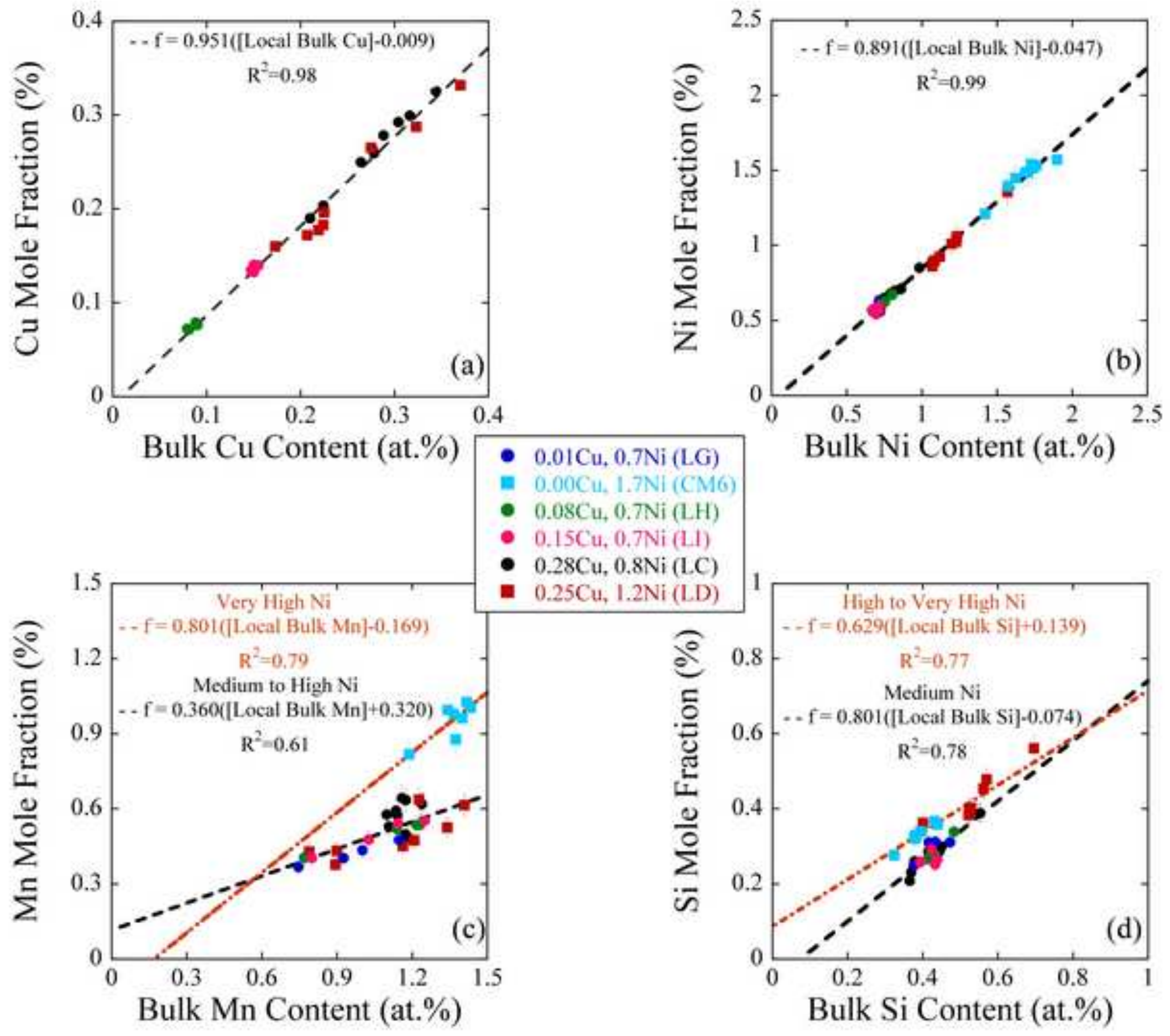




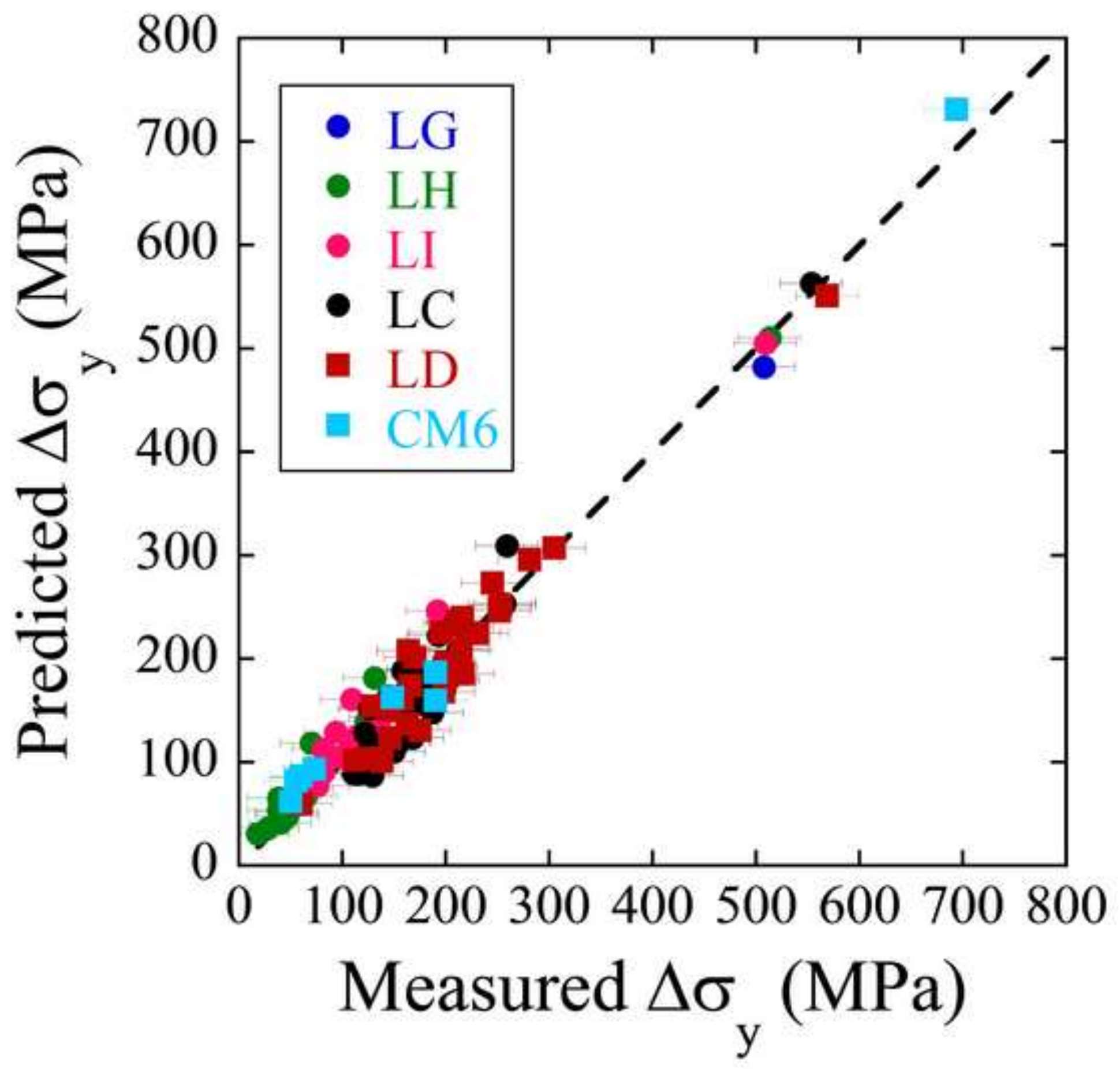


Table 1. Irradiation Conditions

\begin{tabular}{|c|c|c|c|}
\hline Irradiation & Neutron Flux $\left(\mathrm{n} / \mathrm{cm}^{2}-\mathrm{s}\right)^{*}$ & Neutron Fluence $\left(\mathrm{n} / \mathrm{cm}^{2}\right)^{*}$ & Irradiation Temperature $\left({ }^{\circ} \mathrm{C}\right)$ \\
\hline BR2 & $1.0 \times 10^{14}$ & $1.3 \times 10^{20}$ & 300 \\
\hline ATR & $2.3 \times 10^{14}$ & $1.1 \times 10^{21}$ & 290 \\
\hline${ }^{*} \mathrm{E}>1 \mathrm{MeV}$ & & & \\
\hline
\end{tabular}


Table 2. Nominal steel compositions (wt.\%).

\begin{tabular}{|l|l|l|l|l|l|l|l|l|}
\hline Alloy & $\mathrm{Cu}$ & $\mathrm{Ni}$ & $\mathrm{Mn}$ & $\mathrm{Mo}$ & $\mathrm{P}$ & $\mathrm{C}$ & $\mathrm{Si}$ & $\mathrm{Fe}$ \\
\hline LC & 0.41 & 0.86 & 1.44 & 0.55 & 0.005 & 0.14 & 0.23 & bal. \\
\hline LD & 0.38 & 1.25 & 1.38 & 0.55 & 0.005 & 0.19 & 0.23 & bal. \\
\hline LG & 0.01 & 0.74 & 1.37 & 0.55 & 0.005 & 0.16 & 0.22 & bal. \\
\hline LH & 0.11 & 0.74 & 1.39 & 0.55 & 0.005 & 0.16 & 0.24 & bal. \\
\hline LI & 0.20 & 0.74 & 1.37 & 0.55 & 0.005 & 0.16 & 0.24 & bal. \\
\hline CM6 & 0.02 & 1.68 & 1.50 & 0.54 & 0.007 & 0.15 & 0.17 & bal. \\
\hline
\end{tabular}

Also contains some $\mathrm{Cr}, \mathrm{S}$ and other trace elements. L-series heat treatment: austenized at $900^{\circ} \mathrm{C}$ for 1 hour, air cooled, tempered at $664^{\circ} \mathrm{C}$ for 4 hours, air cooled, stress relieved at $600^{\circ} \mathrm{C}$ for 40 hours,

followed by a furnace cooling to $300^{\circ} \mathrm{C}$, then air cooled to room temperature $\mathrm{CM}$ heat treatment: tempered at $660^{\circ} \mathrm{C}$ for 4 hours, air cooled, then stress relieved at $607^{\circ} \mathrm{C}$ for 24 hours followed by a slow

cool at $8^{\circ} \mathrm{C} / \mathrm{hr}$ to $300^{\circ} \mathrm{C}$, then air cooled. 
Table 3. Bulk, nominal and matrix APT compositions for the high fluence condition (G1) (at.\%)

\begin{tabular}{|l|l|l|l|l|l|l|l|l|l|l|l|l|l|l|l|l|l|l|}
\hline & \multicolumn{4}{|l}{ Bulk and Nominal (in parenthesis) Composition (at.\%) } & \multicolumn{4}{l|}{ Matrix Composition (at.\%) } \\
\hline Alloy & $\mathrm{Cu}$ & $+/-$ & $\mathrm{Ni}$ & $+/-$ & $\mathrm{Mn}$ & $+/-$ & $\mathrm{Si}$ & $+/-$ & $\mathrm{Cu}$ & $+/-$ & $\mathrm{Ni}$ & $+/-$ & $\mathrm{Mn}$ & $+/-$ & $\mathrm{Si}$ & $+/-$ \\
\hline LC* & $0.21(0.36)$ & 0.02 & $0.91(0.81)$ & 0.10 & $0.97(1.46)$ & 0.20 & $0.53(0.46)$ & 0.06 & 0.06 & 0.01 & 0.70 & 0.08 & 0.84 & 0.18 & 0.43 & 0.06 \\
\hline LD & $0.21(0.33)$ & 0.01 & $1.00(1.16)$ & 0.01 & $1.08(1.37)$ & 0.10 & $0.52(0.45)$ & 0.03 & 0.06 & 0.00 & 0.74 & 0.02 & 0.91 & 0.08 & 0.41 & 0.03 \\
\hline LG & $0.01(0.01)$ & 0.00 & $0.86(0.69)$ & 0.05 & $1.09(1.36)$ & 0.09 & $0.49(0.43)$ & 0.02 & 0.01 & 0.00 & 0.81 & 0.04 & 1.07 & 0.09 & 0.46 & 0.02 \\
\hline LH & $0.08(0.09)$ & 0.00 & $0.72(0.69)$ & 0.01 & $0.97(1.38)$ & 0.03 & $0.45(0.47)$ & 0.01 & 0.06 & 0.01 & 0.66 & 0.01 & 0.93 & 0.03 & 0.41 & 0.01 \\
\hline LI & $0.15(0.17)$ & 0.01 & $0.72(0.69)$ & 0.01 & $1.21(1.36)$ & 0.03 & $0.43(0.46)$ & 0.01 & 0.06 & 0.00 & 0.59 & 0.00 & 1.11 & 0.02 & 0.37 & 0.01 \\
\hline CM6* & $0.00(0.02)$ & 0.01 & $1.34(1.57)$ & 0.10 & $1.09(1.50)$ & 0.20 & $0.33(0.33)$ & 0.06 & 0.00 & 0.01 & 1.27 & 0.08 & 1.05 & 0.18 & 0.31 & 0.06 \\
\hline
\end{tabular}

*Note that measurements for LC and CM6 from this irradiation condition came from a single APT run of LC and CM6. The uncertainty given for these alloys is twice the largest uncertainty of the other alloys. 
Table 4. Bulk, nominal and matrix APT compositions for the very high fluence condition (ATR1) (at.\%)

\begin{tabular}{|l|l|l|l|l|l|l|l|l|l|l|l|l|l|l|l|l|l|l|}
\hline & \multicolumn{19}{l|}{ Bulk and Nominal (in parenthesis) Composition (at.\%) } \\
\hline Alloy & $\mathrm{Cu}$ & $+/-$ & $\mathrm{Ni}$ & $+/-$ & $\mathrm{Mn}$ & $+/-$ & $\mathrm{Si}$ & $+/-$ & $\mathrm{Cu}$ & $+/-$ & $\mathrm{Ni}$ & $+/-$ & $\mathrm{Mn}$ & $+/-$ & $\mathrm{Si}$ & $+/-$ \\
\hline LC & $0.28(0.36)$ & 0.01 & $0.80(0.81)$ & 0.03 & $1.16(1.46)$ & 0.01 & $0.43(0.46)$ & 0.02 & 0.02 & 0.01 & 0.11 & 0.01 & 0.47 & 0.02 & 0.10 & 0.01 \\
\hline LD & $0.25(0.33)$ & 0.02 & $1.18(1.16)$ & 0.05 & $1.08(1.37)$ & 0.08 & $0.54(0.45)$ & 0.02 & 0.03 & 0.01 & 0.18 & 0.01 & 0.57 & 0.07 & 0.08 & 0.01 \\
\hline LG & $0.00(0.01)$ & 0.01 & $0.71(0.69)$ & 0.01 & $0.87(1.36)$ & 0.08 & $0.43(0.43)$ & 0.01 & 0.00 & 0.00 & 0.08 & 0.01 & 0.37 & 0.06 & 0.10 & 0.01 \\
\hline LH & $0.08(0.09)$ & 0.01 & $0.73(0.69)$ & 0.02 & $1.19(1.38)$ & 0.06 & $0.42(0.47)$ & 0.01 & 0.02 & 0.01 & 0.11 & 0.01 & 0.57 & 0.04 & 0.11 & 0.01 \\
\hline LI & $0.15(0.17)$ & 0.01 & $0.70(0.69)$ & 0.01 & $0.97(1.36)$ & 0.10 & $0.42(0.46)$ & 0.01 & 0.02 & 0.01 & 0.11 & 0.01 & 0.41 & 0.05 & 0.11 & 0.01 \\
\hline CM6 & $0.00(0.02)$ & 0.01 & $1.69(1.57)$ & 0.04 & $1.42(1.50)$ & 0.03 & $0.39(0.33)$ & 0.01 & 0.00 & 0.00 & 0.17 & 0.01 & 0.32 & 0.01 & 0.04 & 0.01 \\
\hline
\end{tabular}


Table 5. Precipitate compositions and $\langle\mathrm{r}\rangle, \mathrm{N}$ and $\mathrm{f}$ at high fluence $(\mathrm{G} 1)$

\begin{tabular}{|c|c|c|c|c|c|c|c|c|c|c|c|c|c|c|c|}
\hline \multirow{2}{*}{ Alloy } & \multicolumn{8}{|c|}{ Precipitate Relative Compositions (at.\%) } & \multirow{2}{*}{ Nominal $\mathrm{Fe}^{* *}$} & \multicolumn{6}{|c|}{$<\mathrm{r}>(\mathrm{nm}), \mathrm{N}\left(\mathrm{m}^{-3}\right), \mathrm{f}(\%)$} \\
\hline & $\mathrm{Cu}$ & $+/-$ & $\mathrm{Ni}$ & $+/-$ & $\mathrm{Mn}$ & $+/-$ & $\mathrm{Si}$ & $+/-$ & & $<\mathrm{r}>$ & $+/-$ & $\mathrm{N}$ & $+/-$ & $\mathrm{f}$ & $+/-$ \\
\hline $\mathrm{LC}^{*}$ & 25.7 & 1.4 & 35.1 & 5.0 & 23.6 & 7.2 & 15.7 & 3.8 & 60.8 & 1.15 & 0.10 & $9.2 \mathrm{E}+23$ & $1.4 \mathrm{E}+23$ & 0.58 & 0.08 \\
\hline $\mathrm{LD}$ & 22.2 & 0.7 & 37.8 & 0.5 & 24.6 & 1.2 & 15.5 & 0.7 & 58.6 & 1.13 & 0.02 & $1.15 \mathrm{E}+24$ & $7 \mathrm{E}+22$ & 0.68 & 0.04 \\
\hline LG & 0.5 & 0.1 & 46.8 & 2.3 & 25.5 & 3.6 & 27.2 & 1.9 & 53.3 & 0.72 & 0.04 & $5.3 \mathrm{E}+23$ & $1.5 \mathrm{E}+23$ & 0.08 & 0.02 \\
\hline $\mathrm{LH}$ & 14.8 & 0.9 & 39.8 & 2.5 & 25.1 & 1.8 & 20.3 & 0.1 & 58.7 & 0.92 & 0.05 & $4.9 \mathrm{E}+23$ & $5 \mathrm{E}+22$ & 0.16 & 0.01 \\
\hline LI & 24.2 & 1.0 & 34.2 & 0.5 & 27.4 & 0.8 & 14.2 & 0.4 & 57.2 & 1.10 & 0.03 & $6.9 \mathrm{E}+23$ & $6 \mathrm{E}+22$ & 0.37 & 0.04 \\
\hline CM6* & 0.1 & 0.1 & 58.9 & 5.0 & 25.6 & 7.2 & 15.5 & 3.8 & 49.6 & 0.70 & 0.10 & $4.6 \mathrm{E}+23$ & $3.0 \mathrm{E}+23$ & 0.07 & 0.04 \\
\hline
\end{tabular}

*Note that measurements for LC and CM6 from this irradiation condition came from a single APT run of LC and CM6. The uncertainty given for these alloys is twice the largest uncertainty of the other alloys. The CM6 mole fraction uncertainty was twice the uncertainty of the LG volume fraction as they had similar measured $\mathrm{f}$.

** Assumed to be an artifact, but provided for alternative interpretations. 
Table 6. Precipitate compositions and $\langle\mathrm{r}\rangle, \mathrm{N}$ and $\mathrm{f}$ at very high fluence (ATR1)

\begin{tabular}{|c|c|c|c|c|c|c|c|c|c|c|c|c|c|c|c|}
\hline \multirow{2}{*}{ Alloy } & \multicolumn{8}{|c|}{ Precipitate Relative Compositions (at.\%) } & \multirow{2}{*}{ Nominal Fe* } & \multicolumn{6}{|c|}{$\left\langle\mathrm{r}>(\mathrm{nm}), \mathrm{N}\left(\mathrm{m}^{-3}\right), \mathrm{f}(\%)\right.$} \\
\hline & $\mathrm{Cu}$ & $+/-$ & $\mathrm{Ni}$ & $+/-$ & $\mathrm{Mn}$ & $+/-$ & $\mathrm{Si}$ & $+/-$ & & $<\mathrm{r}>$ & $+/-$ & $\mathrm{N}$ & $+/-$ & $\mathrm{f}$ & $+/-$ \\
\hline LC & 14.8 & 2.5 & 37.4 & 1.8 & 31.9 & 1.2 & 15.9 & 1.4 & 62.0 & 1.44 & 0.07 & $1.41 \mathrm{E}+24$ & $2.6 \mathrm{E}+23$ & 1.81 & 0.19 \\
\hline $\mathrm{LD}$ & 10.3 & 2.4 & 46.9 & 3.6 & 22.5 & 2.5 & 20.3 & 1.8 & 55.4 & 1.96 & 0.14 & $7.3 \mathrm{E}+23$ & $1.0 \mathrm{E}+23$ & 2.11 & 0.23 \\
\hline LG & 0.2 & 0.1 & 46.1 & 1.5 & 31.2 & 2.8 & 22.5 & 1.6 & 63.5 & 1.25 & 0.04 & $1.63 \mathrm{E}+24$ & $1.6 \mathrm{E}+23$ & 1.33 & 0.03 \\
\hline LH & 5.0 & 0.2 & 40.7 & 1.5 & 35.6 & 2.4 & 18.6 & 1.1 & 64.9 & 1.33 & 0.05 & $1.52 \mathrm{E}+24$ & $2.4 \mathrm{E}+23$ & 1.48 & 0.03 \\
\hline LI & 9.5 & 0.5 & 39.7 & 2.5 & 31.9 & 4.2 & 18.9 & 1.9 & 62.7 & 1.34 & 0.06 & $1.46 \mathrm{E}+24$ & $1.4 \mathrm{E}+23$ & 1.46 & 0.04 \\
\hline CM6 & 0.1 & 0.0 & 52.5 & 1.2 & 35.5 & 1.9 & 11.9 & 0.5 & 58.9 & 1.52 & 0.06 & $1.95 \mathrm{E}+24$ & $1.5 E+23$ & 2.82 & 0.14 \\
\hline
\end{tabular}

* Assumed to be an artifact, but provided for alternative interpretations. 